\title{
Component Structure of Individual Differences in True and False Recognition of Faces
}

\author{
James C. Bartlett, Kalyan K. Shastri, \\ and Hervé Abdi \\ University of Texas at Dallas
}

\author{
Marsha Neville-Smith \\ Texas Woman's University
}

\begin{abstract}
Principal-component analyses of 4 face-recognition studies uncovered 2 independent components. The first component was strongly related to false-alarm errors with new faces as well as to facial "conjunctions" that recombine features of previously studied faces. The second component was strongly related to hits as well as to the conjunction/new difference in false-alarm errors. The pattern of loadings on both components was impressively invariant across the experiments, which differed in age range of participants, stimulus set, list length, facial orientation, and the presence versus absence of familiarized lures along with conjunction and entirely new lures in the recognition test. Taken together, the findings show that neither component was exclusively related to discrimination, criterion, configural processing, featural processing, context recollection, or familiarity. Rather, the data are consistent with a neuropsychological model that distinguishes frontal and occipitotemporal contributions to face recognition memory. Within the framework of the model, findings showed that frontal and occipitotemporal contributions are discernible from the pattern of individual differences in behavioral performance among healthy young adults.
\end{abstract}

Keywords: face recognition, false recognition, individual differences, recognition memory, principalcomponents analysis

False memory phenomena are receiving much attention (Brainerd \& Reyna, 2005), but the voluminous research on this topic has been limited in its scope. First, most work in this area has focused on words or easily verbalized stimuli. Less attention has been paid to hard-to-verbalize stimuli, such as human faces. False memories for faces are ecologically important when they occur in eyewitness identification (Charman \& Wells, 2007; Penrod \& Bornstein, 2007), and they are relevant to theories of memory because faces and verbal stimuli may be processed and represented in different ways (McKone, Kanwisher, \& Duchaine, 2007; Tanaka \& Farah, 1993, 2003). Second, individual differences in false recognition are rarely addressed in current research. This is unfortunate, because, as has been pointed out repeatedly, examining the pattern of individual differences can aid in "deciphering the basic structure and processes of memory" (Bors \& MacLeod, 1996, p. 436; see also Melton, 1967; Underwood, 1975).

In order to improve our understanding of false facial recognition, we used principal-components analysis (PCA; e.g., Jolliffe, 2002) to analyze individual differences in correct and false recognition of faces. In the experiments reported here, study lists of unfamiliar faces were followed by recognition tests that included

James C. Bartlett, Kalyan K. Shastri, and Hervé Abdi, School of Behavioral and Brain Sciences, University of Texas at Dallas; Marsha Neville-Smith, School of Occupational Therapy, Texas Woman's University.

We thank Catherine Truxillo for help in constructing the stimuli used in Experiments 1 and 2 .

Correspondence concerning this article should be addressed to James C. Bartlett, School of Behavioral and Brain Sciences, University of Texas at Dallas, Campus Mailbox GR41, Richardson, TX 75080-3021. E-mail: jbartlet@utdallas.edu old faces, entirely new faces, and one or two types of difficult lure known to elicit frequent false recognitions. PCAs performed on the recognition data revealed two orthogonal components that consistently differed in their relations to hits and false alarms with the different lure types: Whereas one component was related to false alarms but not to hits, the other was primarily related to hits and to the pattern of false alarms across different lure types. The theoretical importance of these components is best viewed in light of three lines of prior work that we review in turn: The first line involves the lineup task, the second concerns memory impairments linked to frontal-lobe damage and to aging, and the third concerns the "conjunction effect," a well-established phenomenon of false recognition that is robust with faces.

\section{Sequential Presentation of Lineups}

False identifications in the lineup task are affected by a number of variables (Kassin, Tubb, Hosch, \& Memon, 2001; Penrod \& Bornstein, 2007), with presentation format being particularly potent. For example, compared to the standard procedure of presenting all lineup faces simultaneously, presenting lineup faces sequentially reduces false identifications in "target-absent" lineups (i.e., when all faces are foils). It should be noted that sequential presentation has this effect without reducing correct identifications in "target-present" lineups (Lindsay \& Wells, 1985; for a similar asymmetric effect produced by "unbiased" or "fair" lineup instructions, see Steblay, 1997). The effect is important, but the attractiveness of the sequential procedure to the forensic community has been brought into question by several recent studies in which sequential presentation reduced correct identifications in targetpresent lineups as well as false identifications in target-absent 
lineups (e.g., Memon \& Bartlett, 2002; for a review and metaanalysis, see Steblay, Dysart, Fulero, \& Lindsay, 2001). We need to know more about when and how a variable such as sequential presentation might influence false alarms without affecting hits. Face recognition deficits by persons with brain damage and older adults are informative in this regard, and we turn to these deficits next.

\section{Face Recognition in Neuropsychological Patients and} Healthy Older Adults

It is widely known that damage to the right occipitotemporal brain region is linked to deficits in face recognition memory (Damasio, Tranel, \& Damasio, 1990; De Renzi, Perani, Carlesimo, Silveri, \& Fazio, 1994;Milner, 1968; Sergent \& Signoret, 1992; Tovée \& Cohen-Tovée, 1993). It is less widely known that right frontal lobe damage is linked to face-recognition deficits as well (see, e.g., Rapcsak et al., 2001; Rapcsak, Reminger, Glisky, Kaszniak, \& Comer, 1999). It is important that deficits due to right occipitotemporal damage differ qualitatively from deficits due to frontal damage in that the former involve decreased correct recognition whereas the latter involve increased false recognition. For example, in tests of old/new recognition of previously unfamiliar faces, Rapcsak et al. (1999) found that, compared with healthy normal controls, 5 patients with right medial-temporal lobe damage produced far fewer hits to old faces but only slightly more false alarms to new faces. By contrast, 2 other patients with right frontal lobe damage were comparable with healthy normal controls in hits but showed extremely high false-alarm rates. This latter finding was subsequently confirmed with 10 additional patients with right frontal damage and has been shown to extend to "retrograde" tests that require discrimination between famous and nonfamous faces (Rapcsak et al., 1999, 2001).

Healthy older adults resemble patients with frontal lobe damage in the deficits they show in face recognition. Several studies found that older persons, as compared with young adults, have inflated false-alarm rates but similar hit rates (for reviews, see Bartlett, 1993; Searcy, Bartlett, \& Memon, 1999). These findings extend to the lineup task, in which false identifications from target-absent lineups are usually higher among older eyewitnesses than among younger eyewitnesses but correct identifications from targetpresent lineups generally show little or no age difference (see Bartlett \& Memon, 2007). Because a subset of the elderly appears to suffer deficits in frontal processing (see, e.g., Anderson \& Craik, 2000), these findings along with the neuropsychological evidence suggest that an isolable component of face recognition memory (and perhaps recognition memory in general) works selectively to oppose false alarms.

On the basis of their observations, Rapcsak et al. (1999) proposed a neuropsychological model of face recognition (shown in Figure 1). According to the model, a "frontal executive system" works to reduce false recognition through several processes that include "strategic search," "effortful recollection" of contextual information, monitoring the contents of memory retrieval, and decision-making processes (including the setting of criteria). In addition to describing frontally mediated processes that work to reduce false alarms, Rapcsak et al. (1999) proposed an occipitotemporal component, which they characterized as a "face recognition module." The occipitotemporal component supports recog-

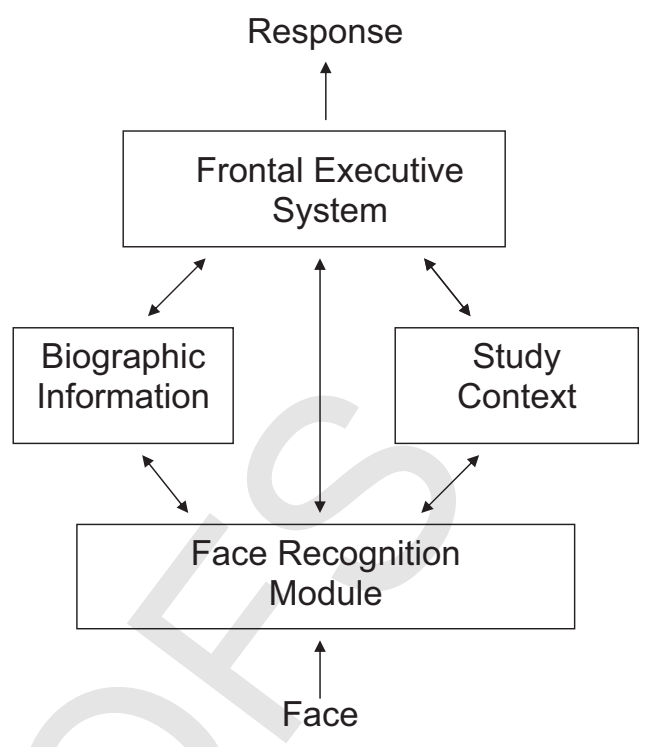

Figure 1. The Rapcsak et al. (1999) neuropsychological model of face recognition. The figure was adapted from Figure 7 of "Neuropsychological Mechanisms of False Facial Recognition Following Frontal Lobe Damage," by S. Z. Rapcsak, S. L. Reminger, E. L. Glisky, A. W. Kaszniak, and J. F. Comer, 1999, Cognitive Neuropsychology, 16, p. 286. Copyright 1999 by Psychology Press. Adapted with permission.

nition of previously viewed faces by signaling resemblance between a face being currently viewed and stored memory representations of previously seen faces.

Although a recognition judgment might be based on a strong resemblance signal, Rapcsak et al. (1999) asserted that "it is only when appropriate contextual information is recovered that we feel confident that a face has been successfully identified" (p. 285; see Mandler, 1980, who first made this point). In the case of wellknown faces, context retrieval refers to the recovery of biographical information. In the case of old/new recognition of previously unfamiliar faces, context retrieval refers to "activating information pertaining to the study episode" (see rightmost box in Figure 1). In either case, context retrieval can occur in two ways. First, it can be triggered in an automatic, bottom-up fashion by the resemblancesignaling face recognition module. Second, it can be retrieved through strategic search and/or effortful recollection controlled by the frontal executive component.

A key claim of the Rapcsak et al. (1999) model is that successful recognition of a previously seen face can occur with minimal executive control through a strong resemblance signal from the face recognition module and/or bottom-up retrieval of contextual and/or biographic information triggered by the output of the module. Hence, successful recognition of previously viewed faces is more dependent on the face recognition module than on the frontal executive component. False recognition of new faces is a different matter. Because all faces share the same first-order configuration (e.g., hairline above eyes, eyes above nose; see Diamond \& Carey, 1986) and faces of different persons can be highly similar in their features, the face recognition module could often signal strong resemblance even for new faces. The model holds that frontally mediated top-down control is critical for reducing false alarms to such faces. 
The Rapcsak et al. (1999) model may require updating (see Rapcsak, 2003, and the General Discussion). Nonetheless, it nicely handles the data on neuropsychology and aging on which it was based and provides a perspective on the question of why, in some conditions, a procedure such as sequential presentation might reduce false recognitions without affecting hits in the lineup task. It also may illuminate a third line of research, to which we turn next.

\section{The Facial Conjunction Effect}

A "conjunction" is a specially constructed recognition-test lure that recombines the parts of two previously studied items. In the case of faces, a conjunction might contain (a) the inner features of one studied face and the outer features of another studied face, as shown in Figure 2 (e.g., Bartlett, Searcy, \& Abdi, 2003); (b) the eyes and mouth of one face and the outer features and nose of another face (see Jones, Bartlett, \& Wade, 2006; Reinitz, Morrissey, \& Demb, 1994); or (c) the eyes and eyebrows of one face with the nose and mouth of another (with hair and other outer features removed; see McKone \& Peh, 2006). On a recognition test that includes old faces, conjunctions, and entirely new faces, hits to old faces are generally more frequent than false alarms to conjunctions. However, the latter are considerably more frequent than false alarms to entirely new faces. For example, hits, conjunction false alarms, and new-face false alarms occurred at rates of approximately $.67, .53$, and .33 , respectively, with upright faces in Experiment 2 of McKone and Peh (2006). The conjunction/new difference in false-alarm errors (.20 in this case) constitutes the conjunction effect. ${ }^{1}$
A

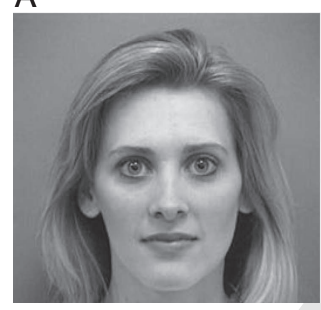

C

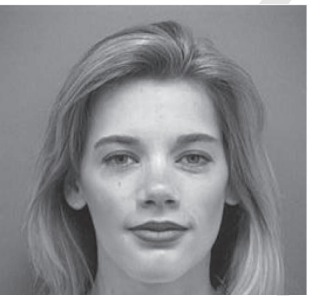

B
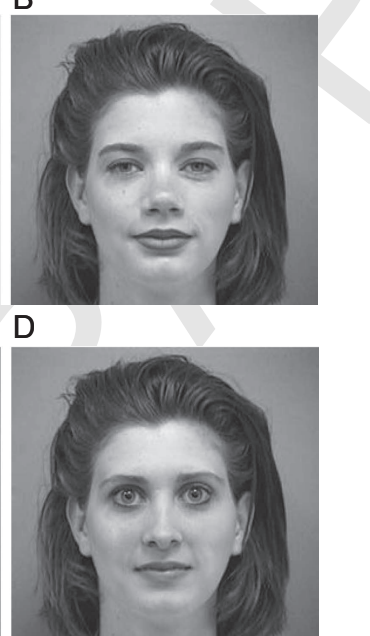

Figure 2. An example of a "quadruplet" with two original faces (A and B) and two synthetic faces (C and D). The synthetics were created by transposing the inner (eyes, nose, and mouth) and outer (hair, ears, and jaw line) facial regions of the originals. Both originals and synthetics served as old and conjunction items in the recognition test (e.g., if a participant had studied faces $\mathrm{C}$ and $\mathrm{D}$, faces $\mathrm{A}$ and $\mathrm{B}$ would serve as conjunctions in the subsequent test). Both individuals gave their written consent for their photographs to be used in research and to be presented as illustrations in scientific publications.
Rapcsak et al. (1999) did not address the conjunction effect in developing their model. Yet, the effect is precisely what one would expect if face recognition involves a component that (a) signals the amount (but not the nature) of resemblance between a currently viewed face and information in memory and (b) can also trigger bottom-up retrieval of contextual information. A facial conjunction is likely to have partial resemblance to the memory representation of each of its two "parents" seen in the study list, such resemblance being based on matching abstract features (e.g., distinctive hookshaped nose) or matching low-level visual codes capturing the gradations in luminance values across facial regions (see Bartlett et al., 2003). In any case, the summed resemblance signal evoked by a conjunction is likely to be strong. A strong resemblance signal may frequently be accompanied by retrieval of contextual information, which can oppose false alarms in some situations (see Kelley \& Jacoby, 2000). However, contextual information is unlikely to be helpful; in the case of conjunctions, it may even be harmful, as the parents of conjunctions were previously studied in the same general context. Hence, the Rapcsak et al. (1999) model correctly predicts that conjunction faces should be highly attractive lures, even if contextual information is frequently retrieved.

\section{The Present Research}

Our goal in the present research was to examine individual differences in correct recognition and false recognition in the facial conjunction paradigm and to determine whether these differences might be explained by the Rapcsak et al. (1999) model or by alternative conceptions of recognition memory. Our point of departure was a PCA of the data from a previously published facialconjunction study (Searcy et al., 1999, Experiment 2). Seventyfive young adults and 76 older adults viewed a study list of 16 photographs of faces; each face was presented twice. This was followed by a test containing 8 old faces, 8 new faces, and 8 conjunctions. Each conjunction combined the internal features (eyes, nose, and mouth) of one study-list face with the external features (hair, ears, jaw, and chin) of another (see Figure 2). The task was to classify old faces as "old" and to reject both conjunctions and new faces as "new." As in prior research with conjunction faces, we found that hit rates for old faces $(M=.78)$ exceeded false-alarm rates for conjunctions $(M=.41)$, which in turn exceeded false-alarm rates for new faces $(M=.14)$.

The PCA was performed on a table ( 3 columns and 151 rows) containing the hit rates for old faces, the false-alarm rates for conjunctions, and the false-alarm rates for new faces for each of

\footnotetext{
${ }^{1}$ The conjunction effect is well established with a variety of stimuli (see Jones \& Jacoby, 2001; Kroll, Knight, Metcalfe, Wolf, \& Tulving, 1996; Reinitz, Lammers, \& Cochran, 1992; Rubin, Van Petten, Glisky, \& Newberg, 1999; Stark \& Squire, 2003), but the effect with faces poses a puzzle because it conflicts with evidence that faces are processed configurally as wholes. McKone and Peh (2006) used simplified faces designed to maximize the role of configural-holistic processing when the faces were upright, and yet they found that the difference between hit rates and conjunction error rates was moderate at best (about .14). Moreover, when the faces were inverted to reduce configural-holistic processing, the conjunction effect (i.e., the conjunction/new difference in false-alarm rates) was not changed (it was about .20, regardless of orientation). The puzzle of the facial conjunction effect remains unsolved.
} 
the 151 participants. It produced two components with eigenvalues greater than 1.0. The upper graph in Figure 3 displays the loadings of each stimulus condition on each of the two components. Note that the first component, which accounted for $42 \%$ of the variance (eigenvalue $=1.25$ ), produced a near-0 loading for hits but strong negative loadings for conjunction false alarms and new-face false alarms. Hereafter, we refer to this first component as the "falsealarm-rate component," or "Factor 1." The second component accounted for $39 \%$ of the variance (eigenvalue $=1.15$ ) and produced a strong positive loading for hits, a moderate positive loading for conjunction false alarms, and a moderate negative loading for new-face false alarms. Hereafter, we refer to this second component as the "hit-rate component," or "Factor 2." The outcome indicated that hits and false alarms in face recognition memory reflect separable processing components, in line with the Rapcsak et al. (1999) model. Beyond this, the PCA suggested that the conjunction effect is linked to the hit-rate component (Factor 2) and not the false-alarm-rate component (Factor 1). ${ }^{2}$

This last point is best illustrated in Figure 4, which shows the mean proportions of "old" judgments made to each of the three item types as a function of Factor 1 scores (left-hand graph) and Factor 2 scores (right-hand graph). ${ }^{3}$ In each case, we sorted the participants by their scores on the factor and then placed them into six different groups ( $n=25$, or 26 per group) on the basis of these factor scores. The lowest scoring group was labeled Group 1, the next-lowest scoring group was labeled Group 2, and so on through

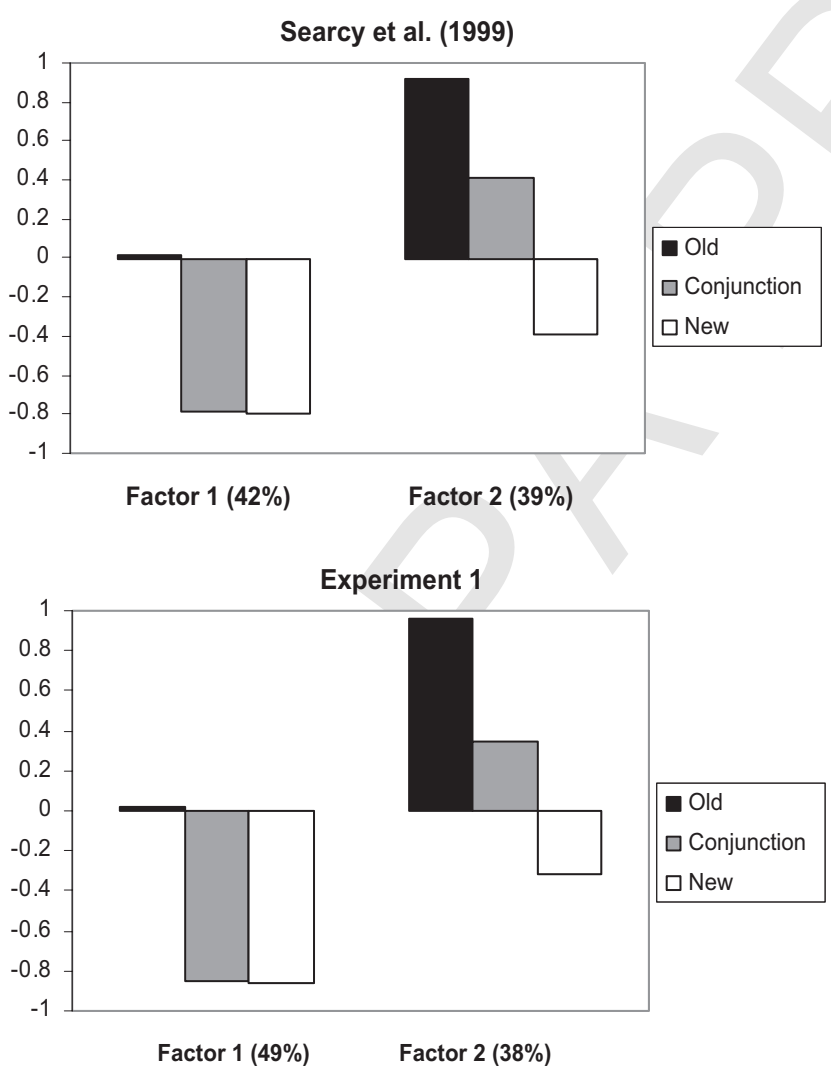

Figure 3. Loadings for hits to old faces and false alarms to conjunction and new faces on Factors 1 and 2 emerging from the PCA of the Searcy et al. (1999) experiment (top) and of Experiment 1 (bottom).

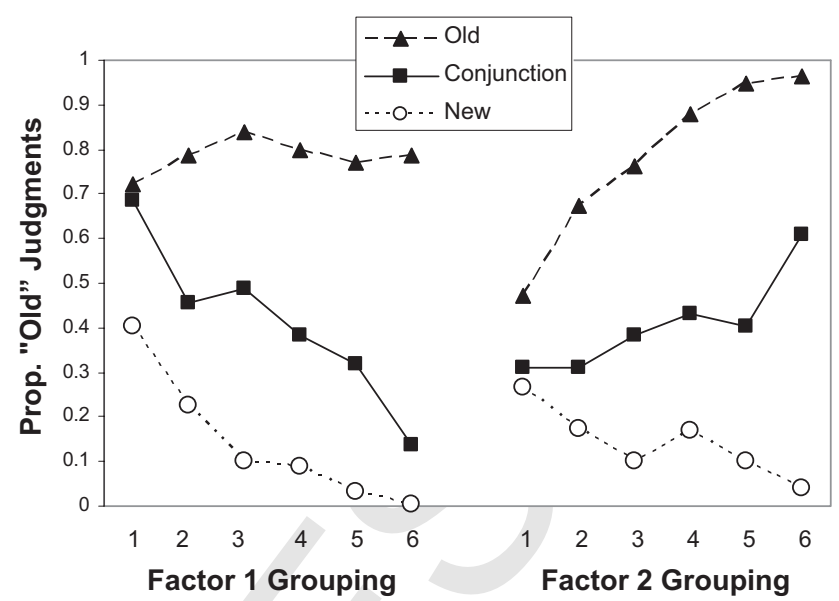

Figure 4. Hit rates for old faces and false-alarm rates for conjunction and new faces in six subsets of participants in the Searcy et al. (1999) study, grouped by their Factor 1 scores (left) and Factor 2 scores (right). Prop. = proportion.

Group 6 (the highest scoring group). Note in the left-hand graph that hits were largely unrelated to Factor 1 scores but that false alarms fell as Factor 1 scores increased. This effect was apparent with both types of lure. By contrast, the right-hand graph shows that hits rose steeply with Factor 2 scores. Furthermore, although conjunction false alarms rose with Factor 2 scores, new-face false alarms decreased. As a result, the conjunction effect (i.e., the conjunction/new difference in false-alarm rates) strengthened from .05 in the lowest Factor 2 group to .57 in the highest Factor 2 group. To statistically evaluate this observation, we computed the correlation between individual participants' Factor 2 scores and the conjunction/new difference in individual participants' falsealarm rates. The correlation was robust, $r(149)=.65, p<.001$. By contrast, the corresponding correlation of Factor 1 scores with the conjunction/new difference was nil, $r(149)=.10, n s$.

In summary, the PCA suggested that two components underlie individual differences in face recognition memory, one that is linked to false recognition of both conjunctions and new items and a second that is linked to hits as well as to the conjunction effect. The outcome was of interest, because the two components appeared to map onto the false-alarm-controlling frontal executive system and the hit-enhancing occipitotemporal face recognition module in the neuropsychological model of Rapcsak et al (1999). That hit rates and the conjunction effect were linked to the same component appeared particularly impressive, as this outcome fit the model's claim that the face recognition module-which is important for hits-signals the amount but not the nature of resemblance between a test face and information in memory.

In the experiments that follow, we sought to replicate and extend our original PCA results and also to characterize the hit and

\footnotetext{
${ }^{2}$ In this and all subsequent PCAs, we assigned signs to loadings such that false alarms in response to new faces load negatively on the factors.

${ }^{3}$ Factor scores reflect the extent to which a participant's data reflect the pattern of the loadings on a factor. Hence, participants with higher Factor 1 scores showed lower false-alarm rates, and those with higher Factor 2 scores showed higher hit rates.
} 
false-alarm components in theoretical terms. It was important to replicate the dual-component outcome with a sample of healthy young adults because the Searcy et al. (1999) study included older adults, and so our first component might simply reflect that elderly participants make more false alarms. If the two-component outcome is assumed to extend to young-only samples, examination of alternative theoretical characterizations of the two components is important. Experiments 1 and 2 addressed two such alternative hypotheses. The first hypothesis was that our two components reflect uncorrelated variance in discrimination and criterion in the sense of signal detection theory (Macmillan \& Creelman, 2005). The data in Figure 4 appear to rule against this notion, as both factors are related to differential responding to old faces versus new faces and conjunctions. However, it was important to gather more definitive data using signal-detection-theory measures, and we did so in Experiment 1.

The second hypothesis was that the two components reflected the separable roles of configural and featural information in facial recognition (e.g., Bartlett et al., 2003; Cabeza \& Kato, 2000; Rakover, 2002). Facial inversion (i.e., presenting faces upside down) impairs configural processing more than it does featural processing (Bartlett \& Searcy, 1993; Freire, Lee, \& Symons, 2000; Searcy \& Bartlett, 1996), and, in recognition memory, it impairs discrimination of old faces from both conjunctions and new items (Bartlett et al., 2003; McKone \& Peh, 2006). Hence, a reasonable hypothesis was that our first component, which had strong samesign loadings for conjunction false alarms and new-face false alarms, reflected configural processing, whereas our second component, which had reversed-sign loadings for the two types of false alarm, reflected featural processing. The clear prediction was that facial inversion should weaken our first component much more than our second. We tested this prediction by presenting all faces upside down to half of the participants in both Experiments 1 and 2 .

\section{Experiments $1 \mathrm{a}$ and $1 \mathrm{~b}$}

Experiment 1a was briefly described in Bartlett et al. (2003), though without the PCA results. Experiment $1 \mathrm{~b}$ has not been published in any form. The two studies were identical in design, and both included between-groups variables (presentation frequency and test delay) that were expected to influence recognition accuracy and to ensure sufficient variance for an informative PCA. The two experiments differed only in that all faces at study and at test were shown upright in Experiment 1a and inverted in Experiment $1 b$.

\section{Method}

Participants. Following procedures approved by the Institutional Review Board of Texas Woman's University, 192 participants were recruited from the university community. Their ages ranged from 18 to 53 years $(M=30$ years). Half of the participants served in Experiment 1a, and the remainder served in Experiment $1 \mathrm{~b}$.

Materials. The materials were made from digitally scanned black-and-white, full-frontal, head-and-shoulders view photographs of 48 young-adult males from the 1965 Texas A\&M University yearbook. All had short-cropped hair, a dark coat, white shirt, and dark tie. All faces were photographed against the same gray background. Pairs of faces that shared such characteristics as approximate age, face shape, and complexion were identified. Each face in a pair served as a parent "original" from which two "synthetic" faces were created with Aldus Photostyler and Corel Photo-Paint. Each synthetic face combined the internal facial region (i.e., eyes, nose, and mouth) of one original face with the external facial region (i.e., hair, ears, and jawline) of the other. The resulting stimulus set comprised 24 "quadruplets," each of which included two original faces and two synthetic faces (see Figure 2 for an example of a quadruplet).

Design and procedure. A 32-item study list (plus 2 filler faces at the start and 2 at the end) was followed by a 48 -item recognition test that included 16 old faces, 16 conjunctions, and 16 entirely new faces. The stimuli were shown on a computer screen for $5 \mathrm{~s}$ each, with an interstimulus interval of $2 \mathrm{~s}$ at study and $3 \mathrm{~s}$ at test. Factorial, between-groups manipulations of presentation frequency (one vs. three study-list presentations) and test delay (10 min vs. $24 \mathrm{hr}$ ) were included in both experiments, with 24 participants receiving each combination.

The study and test lists were constructed such that half the faces in each condition were originals and the rest were synthetics. Most important, half of the "conjunctions" on the recognition test were actually original photographs. They served as conjunctions because their inner and outer features were seen at study, as parts of different faces (the synthetics from their quadruplets). Thus, any subtle difference between the original and synthetic faces, such as a difference in naturalness of appearance, could not be used as a sound basis for recognition judgments.

Following presentation of the study list of faces, participants were asked to make "old" or "new" judgments to faces on the recognition test using a six-point confidence scale (on which $1=$ sure new and $6=$ sure old). Participants were shown examples of conjunction faces and were instructed to reject them as "new."

\section{Results and Discussion}

Face orientation was varied across the two experiments, but, for ease of exposition, we treat inversion as a between-groups variable along with presentation frequency (one vs. three study-list presentations) and test delay (10 min vs. $24 \mathrm{hr}$ ). We first report how inversion, presentation frequency, and test delay affected recognition performance and then turn to the PCA results. Given our large sample $(n=192)$, we set alpha level for all statistical tests at .01.

Hit and false-alarm rates. As shown in Figure 5, the hit rate $\mathbf{F 5}$ for old faces exceeded the false-alarm rate for conjunctions, which in turn exceeded the false-alarm rate for entirely new faces $(M \mathrm{~s}=$ $0.74,0.52$, and 0.29 , respectively). The figure suggests that although hit rates were more sensitive to presentation frequency, false-alarm rates were more sensitive to inversion. Supporting this interpretation, an analysis of variance (ANOVA) revealed a main effect of item type, $F(2,368)=504.3, M S E=0.02, p<.001$; a main effect of inversion, $F(1,184)=46.0, M S E=0.04, p<.001$; and reliable interactions between item type and inversion and item type and presentation frequency, $F(2,368)=68.0, M S E=0.02$, $p<.001$, and $F(2,368)=31.7, M S E=0.02, p<.001$, respectively. Follow-up ANOVAs revealed that hits were affected both by presentation frequency and by inversion, though the frequency effect was considerably stronger, $F(1,184)=39.4, M S E=0.02$, $p<.001$ and $F(1,184)=12.2, M S E=0.02, p<.001$, respectively. Conjunction false alarms were affected by inversion, $F(1$, 


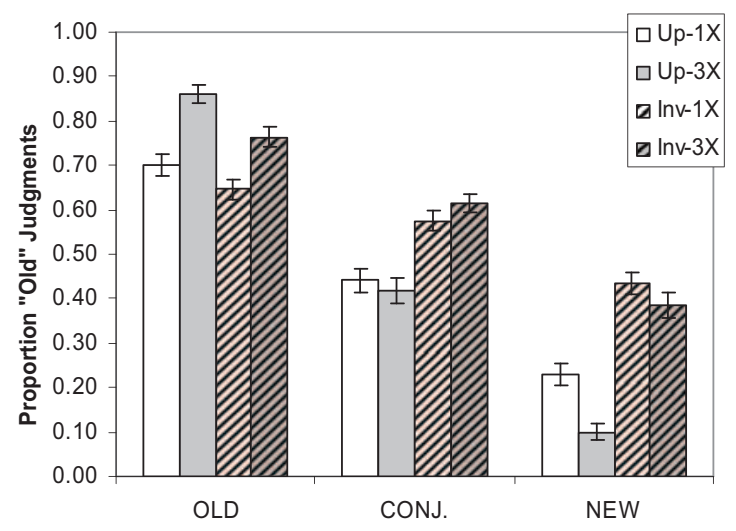

Figure 5. Hit rates for old faces and false-alarm rates for conjunction and new faces for each combination of presentation frequency $(1 \times$ vs. $3 \times)$ and facial orientation (upright [Up] vs. inverted [Inv]). Error bars depict standard errors of the means.

184) $=47.3$, MSE $=0.03, p<.001$, but not by presentation frequency, whereas new-face false alarms were affected by inversion, $F(1,184)=105.5, M S E=0.03, p<.001$, more strongly than by frequency, $F(1,184)=14.2, M S E=0.03, p<.001$. We note that the original ANOVA produced a weak Item Type $\times$ Test Delay interaction, $F(2,368)=5.19, M S E=0.02, p<.01$, but test delay was without reliable effects in the follow-up ANOVAs.

$P C A$. Table 1 shows the intercorrelations among our three basic measures (hit rates for old faces, false-alarm rates for conjunctions, and false-alarm rates for new faces) across the two experiments and between-groups conditions. It also shows the loadings of each measure on each of the two components revealed by the PCA. Except where noted, components are not discussed unless their eigenvalues exceed 1.0. The two components in Table 1 had eigenvalues of 1.46 and 1.15 .

The loadings of our measures on the two components were very similar to those observed in our reanalysis of the Searcy et al. (1999) data (compare top and bottom graphs in Figure 3). As before, Factor 1 showed a near-0 loading for hits, along with strong negative loadings for both types of false alarms. And, once again, Factor 2 showed a strong positive loading for hits, a smaller positive loading for conjunction false alarms, and a negative loading for new-face false alarms.

Figure 6 shows plots of hit and false-alarm rates for eight subsets of 24 participants each, grouped according to their Factor 1 scores (left graph) and Factor 2 scores (right graph). As with the Searcy et al. (1999) data, Factor 1 scores were strongly related to false-alarm errors with both conjunctions and new items, but they were not systematically related to the conjunction/new difference in false alarms. By contrast, Factor 2 scores were strongly related to hits and to the conjunction/new difference in false alarms: This difference rose from .01 in the lowest Factor 2 group to .52 in the highest Factor 2 group. Supporting this observation, we found a robust correlation between individual participants' Factor 2 scores and the conjunction/new difference in individual participants' false-alarm rates, $r(190)=.64, p<.001$, and a near-0 correlation between Factor 1 scores and the conjunction/new difference, $r(190)=.11, n s$.
Factor scores and signal-detection measures. To determine if the PCA components might be characterized as criterion and discrimination, we computed $d^{\prime}$ and $C$ scores (see Macmillan \& Creelman, 2005) from each participant's hit rate for old faces and false-alarm rate for new faces and then computed the correlation of each measure with the participant's Factor 1 and Factor 2 scores. As can be seen in Table 2 (columns 1 and 2), the old/new $d^{\prime}$ scores were T2 positively (and reliably) correlated with Factor 1 and 2 scores (with $d f=190,|r s| \geq 19$ are reliable at $p<.01)$. The $C$ scores were also reliably correlated with both factor scores, though in this case the correlation was positive for Factor 1 and negative for Factor 2. The same pattern was obtained when the $d^{\prime}$ and $C$ scores were derived from hit rates for old faces and false-alarm rates for conjunctions (see Table 2, columns 3 and 4). We conclude that neither component can be characterized as exclusively "criterion" or "discrimination."

Effects of inversion on factor scores. To determine if one of our two components reflected the use of orientation-specific configural processing, we examined the distribution of Factor 1 and Factor 2 scores from the 96 participants from Experiment 1a (upright presentation) and the 96 participants from Experiment 1b (inverted presentation). The outcome is shown in Figure 7, which F7 displays Factor 1 and 2 scores for participants in the onepresentation condition (triangles) and three-presentations conditions (circles) of Experiment 1a (upright faces/filled symbols) and Experiment $1 \mathrm{~b}$ (inverted faces/empty symbols). The large symbols are the mean factor scores for each of the four conditions. The empty symbols generally fall to the left of the filled symbols, indicating that inversion lowered Factor 1 scores. However, the empty symbols also tend to fall lower on the graph than do the filled symbols, indicating that inversion reduced Factor 2 scores as well. ANOVAs confirmed that inversion reduced both Factor 1 scores, $F(1,184)=113.7, M S E=0.61, p<.001$, and Factor 2 scores, $F(1,184)=11.1, M S E=0.79, p<.001$, though the former effect was stronger. By contrast, study-list repetition increased Factor 2 scores, $F(1,184)=39.2, M S E=0.79, p<.001$, without reliably affecting Factor 1 scores at our chosen alpha level of $.01, F(1,184)=4.5, M S E=0.61, p=.04$.

In summary, Experiments $1 \mathrm{a}$ and $1 \mathrm{~b}$ replicated the finding that two separate components underlie performance in the face conjunction paradigm. One component was linked to false alarms (with both conjunctions and new items) and appeared unrelated to the conjunction effect (i.e., the conjunction/new difference in false

Table 1

Correlations Between Proportions of "Old" Judgments for Old, Conjunction, and New Faces and Loadings of Each Measure on Two (Unrotated) Factors Identified by the Principal-Components Analysis (PCA) of Experiment 1

Correlations $(r \mathrm{~s})$ between measures and with factor loadings

\begin{tabular}{lrrr} 
Measure/PCA factor & Old & Conjunction & New \\
\hline Conjunction & .21 & & \\
New & -.22 & .46 & \\
Factor 1 & .02 & -.85 & -.86 \\
Factor 2 & .96 & .35 & -.32 \\
\hline
\end{tabular}



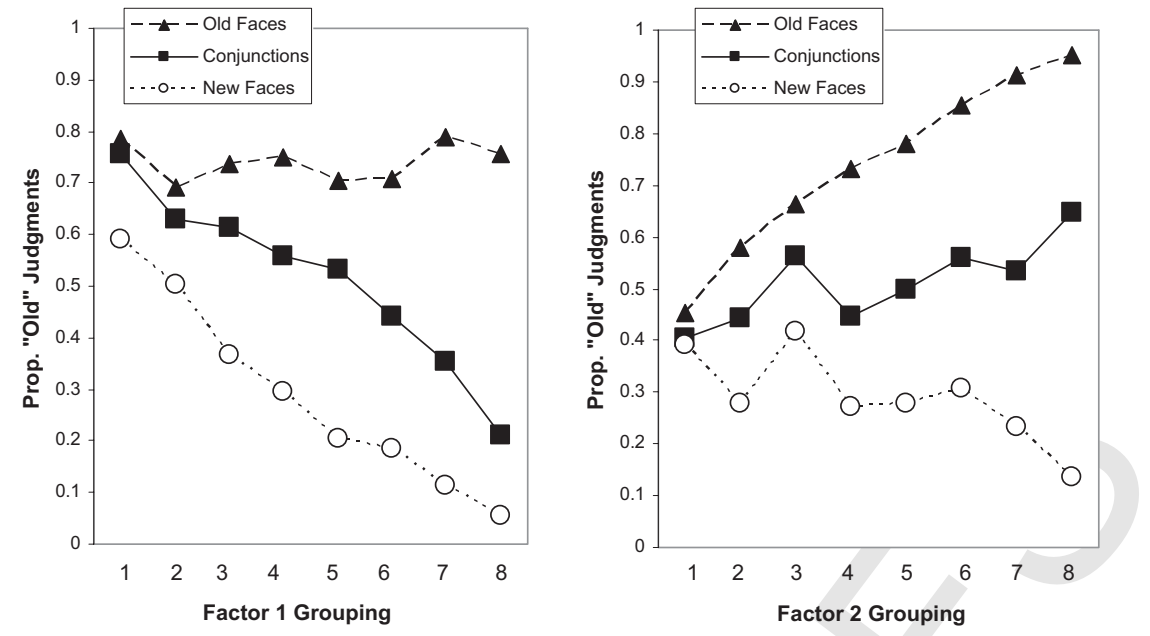

Figure 6. Hit rates for old faces and false-alarm rates for conjunction and new faces in eight subsets of participants in Experiment 1, grouped by their Factor 1 scores (left) and Factor 2 scores (right). Prop. = proportion.

alarms). By contrast, a second component was strongly related to hits as well as to the conjunction effect. Both components were related to discrimination and criterion, and so it is impossible to characterize either component as exclusively discrimination or criterion. Finally, inversion affected Factor 1 scores more strongly than Factor 2 scores. Although this finding might be viewed as supporting the hypothesis that Factor 1 reflects configural processing, the findings of Experiment 2 undermine the hypothesis.

\section{Experiments $2 \mathrm{a}$ and $2 \mathrm{~b}$}

These two experiments further examined the effects of inversion as well as study-list repetitions in the conjunction paradigm (Experiment 2a was briefly described in Bartlett et al., 2003). Participants viewed a study list in which eight faces appeared one time and eight additional faces appeared eight times. The subsequent test list included four old faces that had appeared once at study, four old faces that had appeared eight times at study, four conjunctions whose parents had appeared once at study, and four conjunctions whose parents had appeared eight times at study. It also included eight entirely new faces. Half of the participants in

Table 2

Pearson Product-Moment Correlations Between Factors 1 and 2 of the Principal-Components Analysis (PCA) and d' and $C$ Scores for Old/New Discrimination and for Old/Conjunction Discrimination Across the 192 Participants in Experiment 1

\begin{tabular}{llllll}
\hline & \multicolumn{2}{c}{$\begin{array}{c}\text { Old/new } \\
\text { discrimination }\end{array}$} & & \multicolumn{2}{c}{$\begin{array}{c}\text { Old/conjunction } \\
\text { discrimination }\end{array}$} \\
\cline { 2 - 3 } PCA factor & $d^{\prime}$ & $C$ & & $d^{\prime}$ & $C$ \\
\hline Factor 1 & .61 & .73 & & .65 & .48 \\
Factor 2 & .77 & -.42 & & .44 & -.86 \\
\hline
\end{tabular}

Note. All correlations are reliable $(p<.001)$. each experiment saw all faces upright, and the remainder saw all faces inverted (hence, orientation was a true independent variable).

The experiments assessed the generality of the two-component outcome across two very different levels of learning (one vs. eight presentations at study), and they provided a second look at the effects of inversion on Factors 1 and 2. In addition, Experiment 2a included a manipulation of retroactive interference with which we examined how forgetting might alter Factors 1 and 2. In Experiment $2 b$, we examined whether proximal presentation of the par-

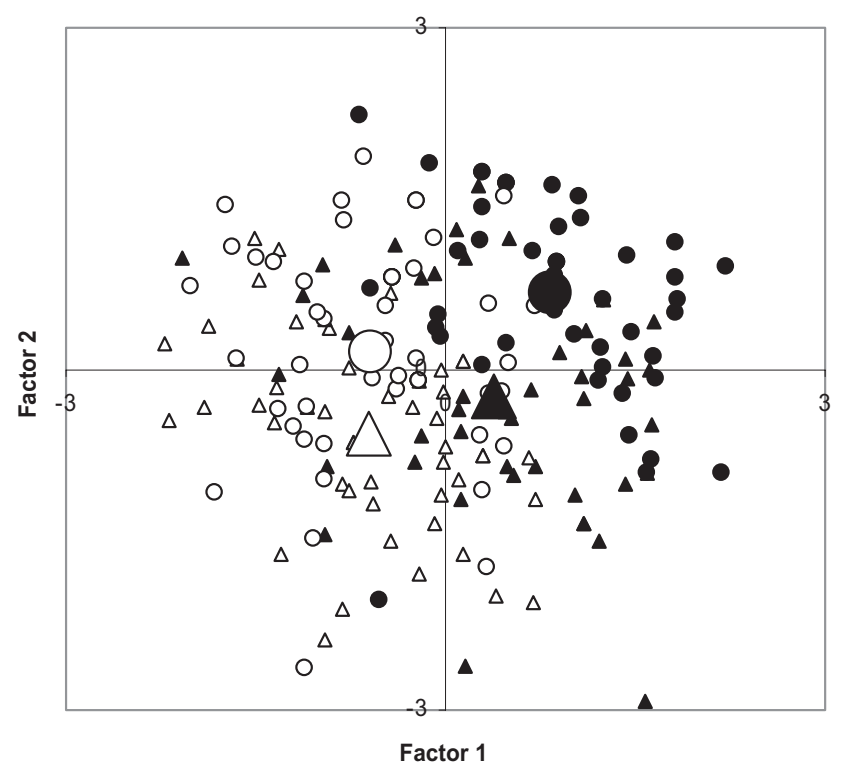

Figure 7. Factor 1 and Factor 2 scores for individual participants who saw the study-list faces one time (triangles) or three times (circles) in Experiment 1a (filled symbols) and Experiment $1 \mathrm{~b}$ (empty symbols). All faces were shown upright in Experiment 1a and inverted in Experiment $1 \mathrm{~b}$. Enlarged symbols represent group means. 
ents of conjunctions might alter the conjunction effect and affect the factor scores.

\section{Method}

The participants in both experiments ( $n \mathrm{~s}=112$ and 96, respectively) were undergraduate students at the University of Texas at Dallas (approximately $67 \%$ female) who participated as one alternative means of fulfilling a course requirement. The stimulus set comprised 12 quadruplets, each of which consisted of 2 original and 2 synthetic faces. The study list included the 2 originals from each of 4 quadruplets and the 2 synthetics from each of 4 additional quadruplets, totaling 16 faces in all. Half of the faces were presented eight times in the study list, with at least 2 faces separating a study-list face and its nearest repetition. The remaining study-list faces were presented just once. The 24-item recognition test included (a) 4 old faces shown one time at study $(1 \times$ -old condition), (b) 4 old faces shown eight times at study $(8 \times$ -old condition), (c) 4 conjunctions whose parents were shown one time at study $(1 \times$-conjunction condition), (d) 4 conjunctions whose parents were shown eight times at study $(8 \times$-conjunction condition), and (e) 8 new faces drawn from 4 quadruplets that were not used at study. Although the faces that appeared in the new condition were the same for all participants, counterbalancing ensured that each of the 16 faces that appeared in the old and conjunction conditions at test served equally often in the $1 \times$-old, $8 \times$-old, $1 \times$-conjunction, and $8 \times$-conjunction conditions.

In each experiment, participants were randomly assigned to one of four experimental groups that were defined by the factorial combination of orientation (all faces shown either upright or inverted) and one additional independent variable. In Experiment $2 \mathrm{a}$, this variable was retroactive interference. Half of the participants viewed an interference set of 48 faces-similar in appear- ance to the study-list faces-immediately following the study list. The other half of the participants took a vocabulary test requiring the same amount of time (6 min).

In Experiment 2b, the independent variable was parent proximity. In the proximal condition, the two study-list faces taken from each quadruplet were always presented in successive list positions. Therefore all the conjunctions in the subsequent test were proximal conjunctions (i.e., their parents has been studied close together). In the nonproximal condition, the two study-list faces from a given quadruplet were never presented in successive list positions, and thus all the conjunctions in the subsequent test were nonproximal conjunctions. Parent proximity can inflate conjunction errors (Hannigan \& Reinitz, 2000; Kroll, Knight, Metcalfe, Wolf, \& Tulving, 1996; McKone \& Peh, 2006; Underwood, Kapelak, \& Malmi, 1976), but this effect is found primarily with simplified face stimuli and when the proximal condition entails either simultaneous (e.g., sideby-side) or alternating (A, B, A, B; C, D, C, D ... ) presentation of the conjunctions' parents (see Jones et al., 2006). The stimuli used here were full-face photographs, and the proximal condition involved merely proximal presentation-not simultaneous or alternating presentation - of the conjunctions' parents. Thus, we did not obtain a standard proximity effect. However, proximity interacted with facial inversion and clarified the relation of configural processing to Factors 1 and 2.

The facial stimuli were viewed on a 50-cm-wide Panasonic video monitor at a distance of approximately $1.5 \mathrm{~m}$. All other aspects of design and procedure were the same as in Experiment 1.

\section{Results and Discussion}

Hit and false-alarm rates. Table 3 displays hit rates for old T3 items presented one versus eight times at study $(1 \times$ - and $8 \times$-old items) along with false-alarm rates for conjunctions whose parents

Table 3

Means and Standard Deviations of Proportions of "Old" Judgments for New Faces, Conjunctions of Faces Studied Once $(1 \times-$ Conj. $)$ and Eight Times $(8 \times-$ Conj. $)$ and of Old Faces Studied Once $(1 \times-$ Old $)$ and Eight Times $(8 \times-$ Old $)$ in the Upright and Inverted Conditions of Experiment $2 a(E 2 a)$ and the Nonproximal and Proximal Conditions of Experiment $2 b$ (E2b-nprox, E2b-prox), Along With Upright-Inverted and Proximal-Nonproximal Differences

\begin{tabular}{|c|c|c|c|c|c|c|c|c|c|c|}
\hline \multirow[b]{3}{*}{ Experiment and condition } & \multicolumn{10}{|c|}{ Item type } \\
\hline & \multicolumn{2}{|c|}{ New } & \multicolumn{2}{|c|}{$1 \times$-conj. } & \multicolumn{2}{|c|}{$8 \times$-conj. } & \multicolumn{2}{|c|}{$1 \times$-old } & \multicolumn{2}{|c|}{$8 \times$-old } \\
\hline & $M$ & $S D$ & $M$ & $S D$ & $M$ & $S D$ & $M$ & $S D$ & $M$ & $S D$ \\
\hline \multicolumn{11}{|l|}{ E2a } \\
\hline Upright & 0.08 & 0.13 & 0.23 & 0.23 & 0.35 & 0.31 & 0.50 & 0.29 & 0.90 & 0.16 \\
\hline Inverted & 0.25 & 0.18 & 0.41 & 0.28 & 0.57 & 0.26 & 0.50 & 0.26 & 0.79 & 0.24 \\
\hline Difference & -0.17 & & -0.18 & & -0.22 & & 0.00 & & 0.11 & \\
\hline \multicolumn{11}{|l|}{ E2b-nprox } \\
\hline Upright & 0.14 & 0.19 & 0.18 & 0.21 & 0.27 & 0.22 & 0.42 & 0.20 & 0.84 & 0.28 \\
\hline Inverted & 0.29 & 0.19 & 0.36 & 0.29 & 0.55 & 0.28 & 0.39 & 0.26 & 0.80 & 0.18 \\
\hline Difference & -0.15 & & -0.19 & & -0.28 & & 0.03 & & 0.04 & \\
\hline \multicolumn{11}{|l|}{ E2b-prox } \\
\hline Upright & 0.14 & 0.20 & 0.36 & 0.22 & 0.52 & 0.32 & 0.68 & 0.32 & 0.97 & 0.08 \\
\hline Inverted & 0.19 & 0.18 & 0.33 & 0.20 & 0.49 & 0.33 & 0.44 & 0.25 & 0.79 & 0.22 \\
\hline Difference & -0.05 & & 0.03 & & 0.03 & & 0.24 & & 0.18 & \\
\hline \multicolumn{11}{|l|}{ E2b: Prox-nprox difference } \\
\hline Upright & 0.00 & & 0.19 & & 0.25 & & 0.26 & & 0.13 & \\
\hline Inverted & -0.10 & & -0.03 & & -0.06 & & 0.05 & & -0.01 & \\
\hline
\end{tabular}

Note. Differences in bold italics are reliable by $t$ test at $p<.01$. 
had been viewed one versus eight times at study $(1 \times-$ and $8 \times$ -conjunctions) and false-alarm rates for entirely new faces. The data from Experiment $2 \mathrm{a}$ are collapsed over the retroactive interference condition (which had minimal effects), but the data from Experiment $2 \mathrm{~b}$ are shown separately for the proximal and nonproximal conditions.

Both hits and false alarms rose with presentation frequency, though the increase from the $1 \times$ condition to the $8 \times$ condition was greater with hits (mean difference $=.36$ ) than with conjunction false alarms (mean difference $=.15$ ). Thus, repetition at study served to strengthen the conjunction effect, but at the same time, it increased discrimination between old faces and conjunctions. ${ }^{4}$ Both of these repetition effects appeared with inverted faces (mean differences $=.35$ and .17 , respectively) and upright faces (mean differences $=.37$ and .12 , respectively).

The effects of parent proximity in Experiment $2 b$ are shown at the bottom of Table 3. As expected, conjunction false alarms were more frequent in the proximal condition than in the nonproximal condition. However, the effect occurred only with upright faces, and, there, the proximal condition showed an increase in hits as well as conjunction false alarms.

A useful perspective on this unexpected pattern can be gained by examining the effects of inversion in Experiment $2 \mathrm{a}$, in the methodologically similar nonproximal condition of Experiment $2 \mathrm{~b}$, and in the proximal condition of Experiment $2 \mathrm{~b}$ (see rows 3, 6 and 9 of Table 3). Note that in the first two cases, the effect of inversion is largely restricted to false-alarm rates: Inversion increased conjunction false alarms as well as new-face false alarms and had minimal effects on hits. By contrast, in the proximal condition of Experiment 2b, inversion largely reduced hits. In light of much evidence that inversion impairs processing of configural information, the pattern suggests that configural information can be used either to reduce false alarms or to increase hits, depending on conditions. The PCA presented below substantiates this claim.

Before turning to the PCA, we note that all preceding observations were statistically supported. An ANOVA of hit rates in Experiment $2 \mathrm{a}$ supported the main effect of presentation frequency, $F(1,108)=114.0, M S E=0.06, p<.001$, and an ANOVA of false-alarm rates supported an effect of inversion, $F(1$, $108)=32.6, M S E=0.09, p<.001$, as well as frequency, $F(2$, $216)=62.4, M S E=0.04, p<.001$, with each pairwise comparison of frequency conditions reliable at $p<.01$ (Tukey's honestly significant difference test). In Experiment 2b, the ANOVAs showed Proximity $\times$ Inversion interactions with both hits, $F(1$, $92)=6.64, M S E=0.05, p<.001$, and false alarms, $F(1,92)=$ $8.84, M S E=0.09, p<.01$. In the nonproximal condition, an ANOVA of hits supported the main effect of presentation frequency, $F(1,46)=71.2, M S E=0.06, p<.001$, and an ANOVA of false-alarm rates supported an effect of inversion, $F(1,46)=$ 19.7, MSE $=0.08, p<.001$, as well as frequency, $F(2,92)=$ 11.7, $M S E=0.04, p<.001$, just as in Experiment 2a. However, in the proximal condition, the ANOVA of hits showed main effects of both inversion, $F(1,46)=18.3, M S E=0.06, p<.001$, and frequency, $F(1,46)=49.0, M S E=0.05, p<.001$, whereas an ANOVA of false alarms showed no effect of inversion, $F(1,46)<$ $1, p<.05$, along with a strong effect of frequency, $F(2,92)=$ 31.7, MSE $=0.04, p<.001$.

$P C A$. Table 4 shows the intercorrelations among our five basic measures (rates of "old" judgments for $1 \times$-old faces,
$8 \times$-old faces, $1 \times$-conjunctions, $8 \times$-conjunctions, and new faces) across the two experiments and between-groups conditions. It also shows the loadings of each measure on each of the two components emerging from the PCA (eigenvalues $=1.88$ and 1.14 for Factors 1 and 2, respectively). Figure 8 displays the factor $\mathbf{F} 8$ loadings in a way that facilitates comparison with the data from Experiment 1. It is clear that the factor loadings for $8 \times$-old faces, $8 \times$-conjunctions, and new faces nicely replicated the pattern that we previously obtained (see Figure 3 ). By comparison, the loadings for $1 \times$-old faces, $1 \times$-conjunctions, and new faces produced a degraded pattern, suggesting that the appearance of the two components depends on some requisite level of learning.

Effects of inversion on factor scores. Figure 9 plots the Factor F9 1 and 2 scores for each participant in both the upright (filled symbols) and inverted (empty symbols) conditions. In general, inversion had stronger effects on Factor 1 scores than on Factor 2 scores, as in Experiment 1. However, in the proximal condition of Experiment $2 b$ (represented by circles), Factor 1 scores were generally low, and inversion affected only Factor 2 scores. ANOVAs confirmed that, in Experiment 2a, inversion affected both Factor 1 scores, $F(1,108)=32.7, M S E=0.81, p<.001$, and Factor 2 scores, $F(1,108)=8.75, M S E=0.80, p<.01$, though the former effect was stronger. By contrast, in Experiment $2 b$, there was an Inversion $\times$ Proximity interaction in Factor 1 scores, $F(1,92)=8.51, M S E=0.88, p<.01$, and, at $p<.05$, in Factor 2 scores as well, $F(1,92)=5.24, M S E=0.88, p<.03$. Follow-up ANOVAs of the Factor 1 scores showed that the inversion effect was reliable in the nonproximal condition, $F(1,46)=15.4$, $M S E=0.75, p<.001$, but not in the proximal condition, $F(1$, 46) $<1, p<.05$. Follow-up ANOVAs of the Factor 2 scores showed precisely the opposite pattern. There, the inversion effect was reliable in the proximal condition, $F(1,46)=20.7, M S E=$ $0.71, p<.001$, but not in the nonproximal condition, $F(1,46)<$ $1, p<.05$. These data show that inversion can affect Factor 1 and/or Factor 2, depending on conditions. The outcome undermines the view that either component is exclusively related to orientation-specific configural processing.

Further evidence on this point comes from two follow-up PCAs, one on the data from the upright condition and another on the data from the inverted condition. Each PCA identified two components with eigenvalues exceeding 1 (1.79 and 1.12 in the upright condition, 1.90 and 1.11 in the inverted condition). Because the pattern of factor loadings was generally degraded in the $1 \times$ condition (see Figure 8), we show only the factor loadings from the $8 \times$ and new-item conditions in Figure 10. It is clear that the F10 two-component outcome obtained in the overall analysis and in prior studies generalizes nicely from upright faces to inverted faces. We conclude that both components can be weakened by inversion, so that neither is uniquely linked to orientation-specific configural information.

\footnotetext{
${ }^{4}$ Presentation frequency had these effects in Experiment 1 as well, although the pattern there was slightly different (repetition increased hits and reduced new-face false alarms without affecting conjunction false alarms; see Figure 5). We suspect that participants in the threepresentations condition of Experiment 1 set higher recognition criteria than did those in the one-presentation condition and that this masked an effect of repetition on conjunction false alarms.
} 


\section{Experiment 3}

Experiments 1 and 2 replicated the two-component outcome of our initial PCA. Further, they established that neither component could be uniquely and invariably linked to discrimination, criterion, or orientation-specific configural processing. In Experiment 3, we asked whether Factors 1 and 2 might be linked to recollection and familiarity, the two core concepts of dual-process theories of recognition memory (e.g., Diana, Reder, Arndt, \& Park, 2006; Jacoby, 1991, 1999; Yonelinas, 2002). By the dual-process account, familiarity is the nonspecific feeling that a stimulus has been encountered before. It varies only in strength and provides no information on the context in which the stimulus was encountered. By contrast, recollection involves conscious retrieval of more detailed information about one's prior encounter of the stimulus and can specify the context of this encounter. There is evidence that familiarity falls from old items to conjunctions and from conjunctions to new items (Jones \& Bartlett, 2009; Jones \& Jacoby, 2001). Thus, it is reasonable to suggest that Factor 2 - which shows a graded pattern of loadings for the three itemtypes-reflects familiarity. It also is plausible that Factor 1 might reflect recollection, but the experiment did not directly test this idea. $^{5}$

To test the dual-process hypothesis for Factors 1 and 2, we introduced into the study another kind of lure, which we refer to as a "familiarized lure" (FL). FLs are test-list faces that were seen previously by participants but outside the context of the study list (e.g., Jennings \& Jacoby, 1997). Because FLs differ from study-list faces primarily with respect to their presentation context, a context-free familiarity component should respond to such lures much as it does to truly old faces. Hence, if Factor 2 reflects context-free familiarity, it should show a strong loading for FL false alarms, in the same direction as the loading for hits.

The FLs in Experiment 3 were exact repetitions of new faces presented several items earlier in the recognition test (cf. Jennings \& Jacoby, 1997). Participants were instructed to reject these FLs as "new." Pilot testing indicated that FL false alarms would be comparable in frequency to conjunction false alarms.

Table 4

Correlations Between Proportions of "Old" Judgments for Old, Conjunction, and New Faces and Loadings of Each Measure on Two (Unrotated) Factors Identified by the Principal-Components Analysis (PCA) of Experiment 2

\begin{tabular}{|c|c|c|c|c|c|}
\hline \multirow{2}{*}{$\begin{array}{l}\text { Measure/PCA } \\
\text { factor }\end{array}$} & \multicolumn{5}{|c|}{$\begin{array}{c}\text { Correlations }(r s) \text { between measures and with } \\
\text { factor loadings }\end{array}$} \\
\hline & $1 \times$-old & $8 \times$-old & $1 \times$-conj. & $8 \times$-conj. & New \\
\hline $8 \times$-old & .05 & & & & \\
\hline $1 \times$-conj. & .17 & .01 & & & \\
\hline $8 \times$-conj. & .20 & .04 & .40 & & \\
\hline New & .09 & -.23 & .42 & .37 & \\
\hline Factor 1 & -.39 & .14 & -.77 & -.75 & -.75 \\
\hline Factor 2 & .47 & .86 & .09 & .19 & -.37 \\
\hline
\end{tabular}

Note. $1 \times$-old $=$ old faces studied once; $8 \times$-old $=$ old faces studied eight times; $1 \times$-conj. $=$ conjunctions of faces studied once; $8 \times$-conj. $=$ conjunctions of faces studied eight times.

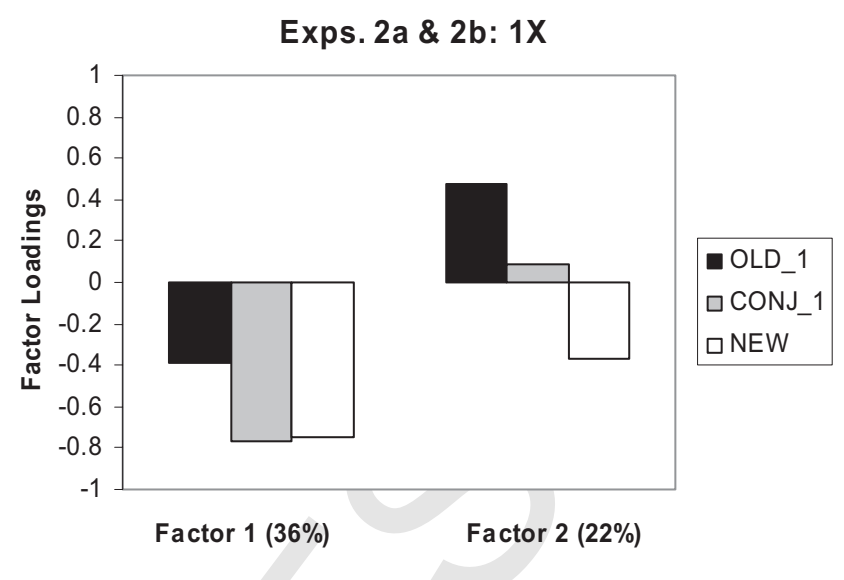

Exps. $2 \mathrm{a} \& 2 \mathrm{~b}: 8 \mathrm{X}$

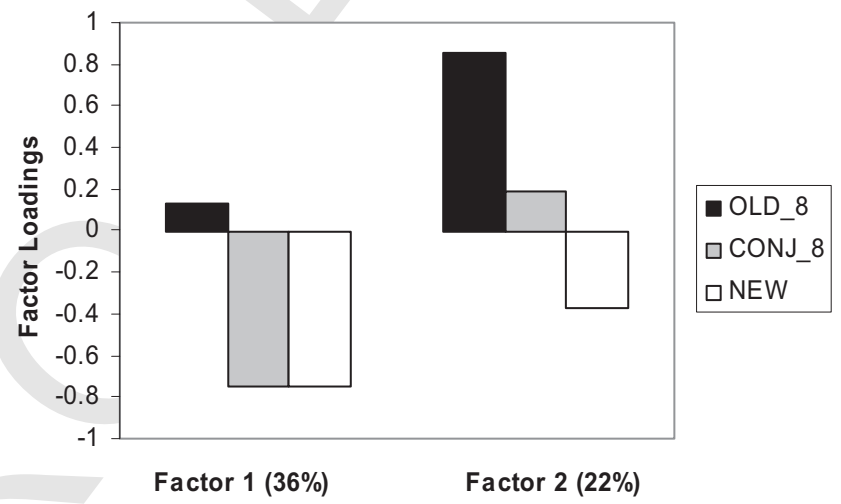

Figure 8. Loadings for hits to old faces and false alarms to conjunction and new faces on Factors 1 and 2 emerging from the principal-components analyses of Experiment 2. The upper panel shows loadings for oncepresented $(1 \times)$ old and conjunction faces, and the lower panel shows loadings for eight-times presented $(8 \times)$ old and conjunction faces. The loadings for new faces are shown in both panels.

\section{Method}

Ninety-six participants were recruited as in Experiment 2. The method was the same as that of Experiment 1 except that (a) the materials were made from a new set of 180 monochromatic, full-frontal-view photographs of faces of male and female students taken 5 or more years before the study (see Figure 2); (b) the study list consisted of 120 faces (plus two fillers at the start and two at the end) and was presented three times, each time in a different randomized sequence; (c) the stimuli were viewed as a PowerPoint slide show on a 46-cm-wide computer monitor, with study-list faces shown for $2 \mathrm{~s}$ each and followed by a 2 -s interstimulus

\footnotetext{
${ }^{5}$ It is now well established that recollection can be used to reject lures as well as to accept targets (Lampinen, Odegard, \& Neuschatz, 2004). Given the near-0 loadings of hit rates on Factor 1, this factor would presumably reflect a "recollection rejection" component rather than a general recollection component.
} 
interval and test-list faces shown for $2 \mathrm{~s}$ each and followed by a 3-s interstimulus interval; and (d) the test list consisted of 240 items, including 60 old faces, 60 conjunctions, 60 new faces, and 60 FLs. ${ }^{6}$ Each FL was an exact repetition of a new face presented 9 to 12 items earlier in the test.

The sequencing of faces in the test list was randomized, with the constraints that (a) each test half included equal numbers of faces in all four conditions and (b) the last new item appeared at least 10 items from the end of the list, so that it was possible to repeat the item (to make a FL) with a lag of at least 9. As in Experiments 1 and 2, participants were instructed to endorse exact matches to study-list items as "old" and to reject all other types of item as "new" using a six-point confidence scale.

\section{Results and Discussion}

Hit and false-alarm rates. The upper panel of Figure 11 displays the hit rates for old faces and false-alarm rates for conjunctions, new faces, and FL faces. As in prior studies, hits were more frequent than conjunction false alarms, which in turn were more frequent than new-face false alarms. FL false alarms occurred at about the same rate as conjunction false alarms. An ANOVA of the proportion of "old" responses produced a strong main effect of item type, $F(3,285)=215.1, M S E=0.02, p<.001$. All pairwise differences were reliable by Tukey's honestly significant difference test except for the difference between conjunctions and FLs.

$P C A$. The upper half of Table 5 displays the intercorrelations among dependent variables along with their loadings on the first two principal components (eigenvalues $=2.19$ and 1.14 , respectively). Figure 12 (upper portion) displays these loadings, along with the mean hit and false-alarm rates for eight subsets of 12 participants when grouped by their Factor 1 scores and by their Factor 2 scores. As in prior studies, Factor 1 emerged as a false-

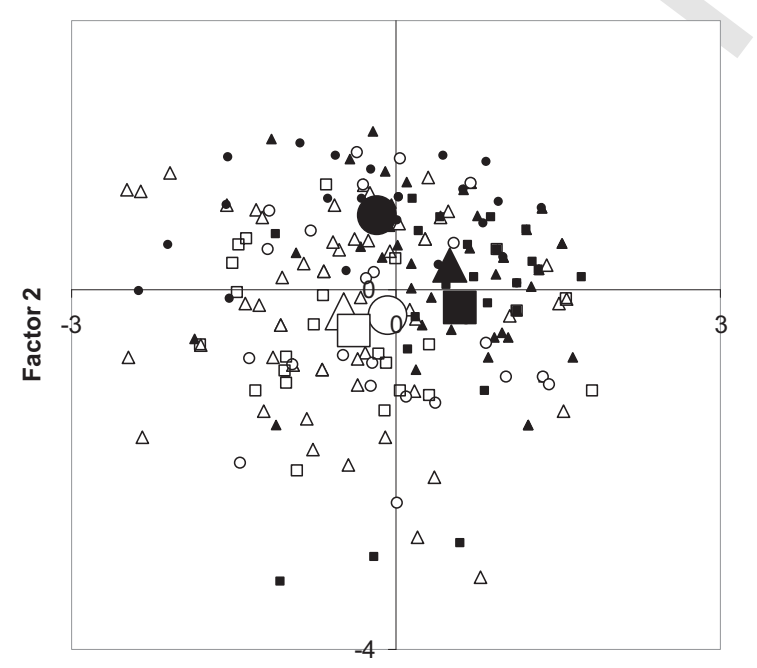

Factor 1

Figure 9. Factor 1 and Factor 2 scores for individual participants in the upright (filled symbols) and inverted (unfilled symbols) conditions of Experiment 2a (triangles) and the nonproximal (squares) and proximal (circles) conditions of Experiment 2b. Enlarged symbols represent group means.

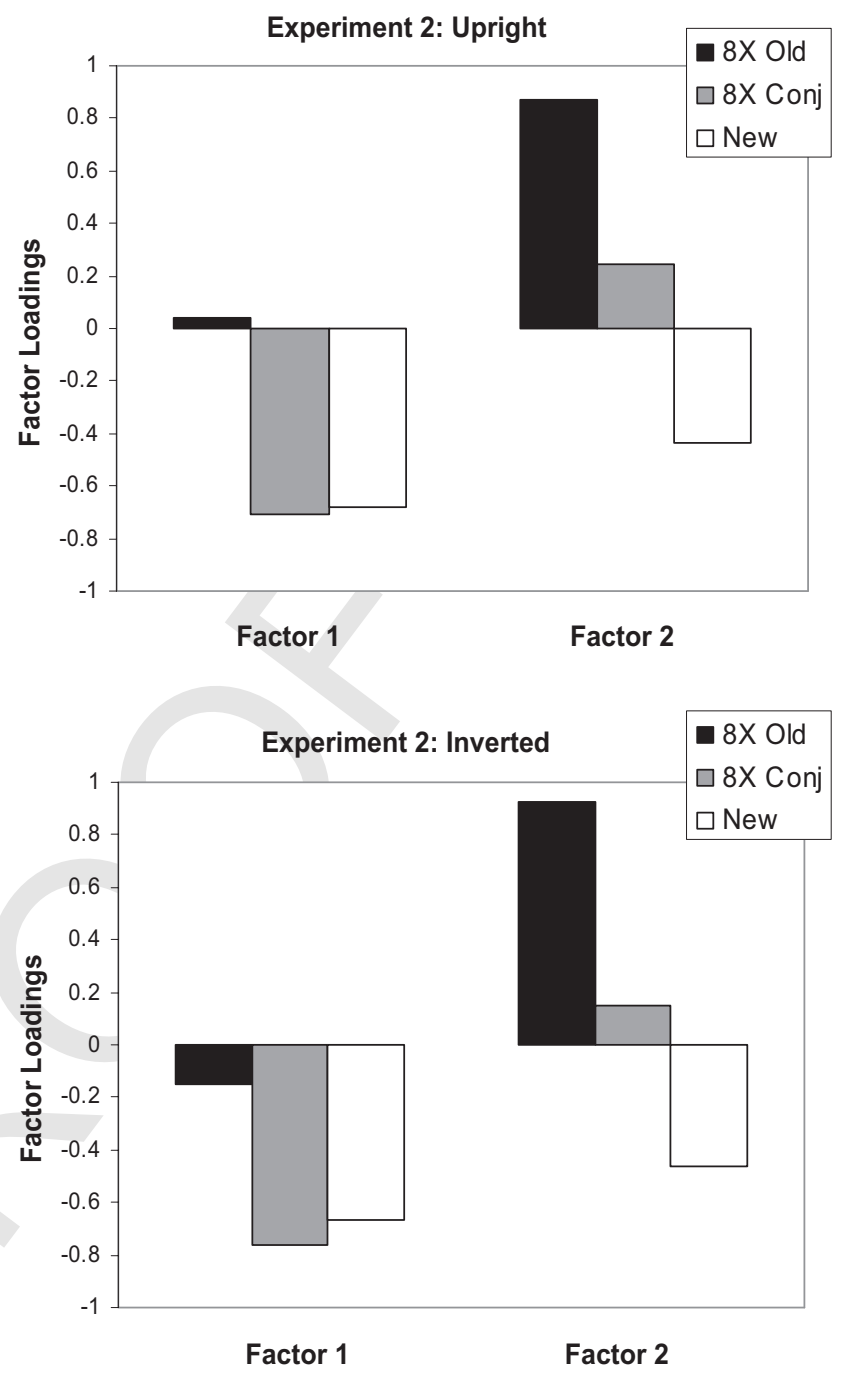

Figure 10. Loadings for hits to old faces and false alarms to conjunction and new faces on Factors 1 and 2 emerging from separate principalcomponents analyses of the upright (top) and inverted (bottom) conditions of Experiment 2. Loadings for old and conjunction (Conj) faces are from the eight-presentations $(8 \times)$ condition only.

alarm-rate component with strong negative loadings for conjunction false alarms $(-.85)$ and new-face false alarms $(-.85)$. Factor 1 also showed a negative loading for FL false alarms (-.76), further supporting its status as a false-alarm-rate component. We note also a moderate negative loading for hits $(-.42)$. This is similar to the Factor 1 hit-rate loading found in the low-learning $(1 \times$-old $)$ condition of Experiment 2 and may reflect the fact that list length was considerably longer in Experiment 3 (120 study-list faces) than in Experiments 1 and 2 (32 and 16 study-list faces, respectively). In any event, the subgroup analysis (upper-right quadrant of Figure 12) shows that only the very highest Factor 1 participants showed an appreciable reduction in hits.

\footnotetext{
${ }^{6}$ The study and test lists were lengthened because we wished this study to be procedurally identical to a parallel, event-related potential study in which many observations per participant and condition were required.
} 
Factor 2 showed a strong loading for hits (.85) and moderate loadings in opposite directions for conjunction false alarms and new-face false alarms (.32 and -.36 , respectively). This result replicated those of Experiments 1 and 2. As in these prior studies, the subgroup analysis (see Figure 12, upper-right quadrant) reveals that Factor 2 scores were strongly related not only to hits but also to the conjunction effect (the conjunction/new difference in falsealarm rates; compare filled squares and empty circles in Figure 12). Again, we quantified the latter relation by computing the correlation between individual participants' Factor 2 scores and the conjunction/new difference in individual participants' false-alarm rates. As before, the correlation was robust, $r(94)=.72, p<.01$.

The most important new finding in Experiment 3 was that Factor 2 showed a loading for FL false alarms $(-.43)$ that was similar to that for new-face false alarms $(-.36)$ and was reversed in sign from that for hits (.85) and conjunction false alarms (.32). As a result, the Factor 2 groupings reveal a striking crisscrossed pattern such that FL false-alarm rates exceeded conjunction falsealarm rates among the lower Factor 2 participants, whereas the reverse was true among higher Factor 2 participants (compare empty circles with filled squares in Figure 12). Note in addition that Factor 2 was quite strongly related to discrimination between
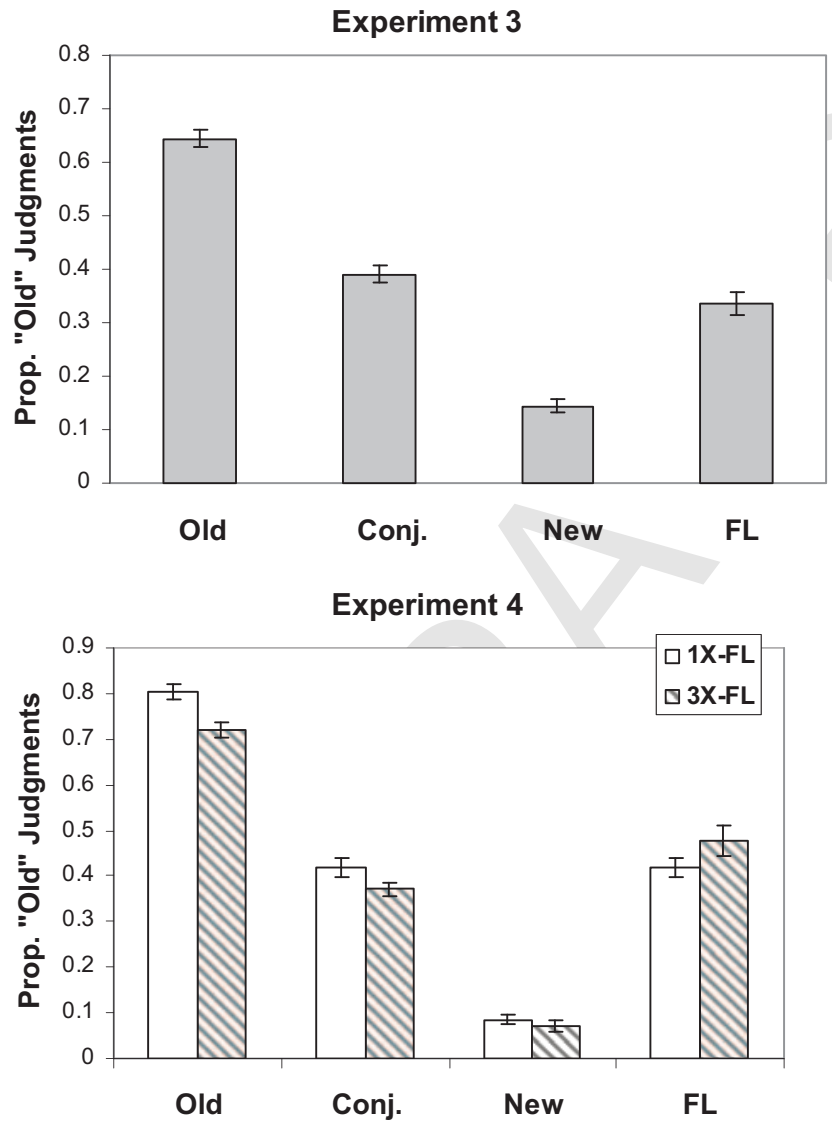

Figure 11. Mean hit rates for old faces and false-alarm rates for conjunctions (Conj.), new faces, and familiarized lures (FL) in Experiment 3 (top) and in Experiment 4 (bottom). $1 \times-\mathrm{FL}$ and $3 \times-\mathrm{FL}$ refer to one versus three presentations of the familiarization set in Experiment 4. Error bars depict standard errors of the means. Prop. = proportion.
Table 5

Correlations Between Proportions of "Old" Judgments for Old, Conjunction, New, and Familiarized Lure (FL) Items and Loadings of Each Measure on Two (Unrotated) Factors Identified by the Principal-Components Analysis (PCA) of Experiment 3 and of Experiment 4

\begin{tabular}{lrrrr}
\hline & \multicolumn{4}{c}{ Correlations $\begin{array}{c}(r \text { s) between measures and with } \\
\text { factor loadings }\end{array}$} \\
\cline { 2 - 5 } $\begin{array}{l}\text { Measure/PCA } \\
\text { factor }\end{array}$ & Old & Conjunction & New & FL \\
\hline Experiment 3 & & & & \\
$\quad$ Conjunction & .50 & & & \\
New & .05 & .59 & .65 & \\
FL & .05 & .40 & -.85 & -.76 \\
Factor 1 & -.42 & -.85 & -.36 & -.43 \\
Factor 2 & .85 & .32 & & \\
Experiment 4 & & & & \\
Conjunction & .32 & .51 & .25 & \\
New & -.01 & .24 & -.80 & -.52 \\
FL & -.15 & -.86 & -.17 & -.59 \\
Factor 1 & -.26 & .25 & \\
Factor 2 & .87 & &
\end{tabular}

old and FL faces, as corroborated by a correlation of $r(94)=.89$, $p<.01$, between individual participants' Factor 2 scores and the differences between individual participants' hit rates for old faces and false-alarm rates for FLs. ${ }^{7}$ This robust correlation contradicts Fn7 the notion that Factor 2 is a context-free familiarity component and suggests that this factor is linked to context retrieval.

\section{Experiment 4}

Although the findings of Experiment 3 weigh against the hypothesis that Factor 2 reflects context-free familiarity, they do so only on the assumption that participants distinguished old and FL faces on the basis of context retrieval. This assumption is plausible, but it is open to question on the grounds that old and FL items may have differed somewhat in familiarity. On the one hand, old faces might have been more familiar than FLs, because old items were shown three times at study and FLs had been shown only one time before (earlier in the test). On the other hand, old faces might have been less familiar than FLs, because FLs were seen more recently ( 9 to 12 items back vs. an average of 180 items back). In either case, participants may have used familiarity information to distinguish old faces from FLs, and, therefore, it might be argued that, after all, Factor 2 reflects familiarity.

To obtain more definitive data, we sought to replicate the findings of Experiment 3 in two conditions, one that allowed a familiarity difference between old faces and FL items and one that was designed to minimize any such difference. The FLs in Experiment 4 were faces viewed in a familiarization set prior to the study list (cf. Jacoby, 1999). This familiarization set was presented only once to half of the participants $(1 \times-\mathrm{FL}$ condition $)$, but it was presented three times to the others $(3 \times-\mathrm{FL}$ condition $)$. The study list was presented three times to all participants. In both condi-

\footnotetext{
${ }^{7}$ The correlation of Factor 2 scores with $d^{\prime}$ s for old/FL discrimination was .87 .
} 
(A)
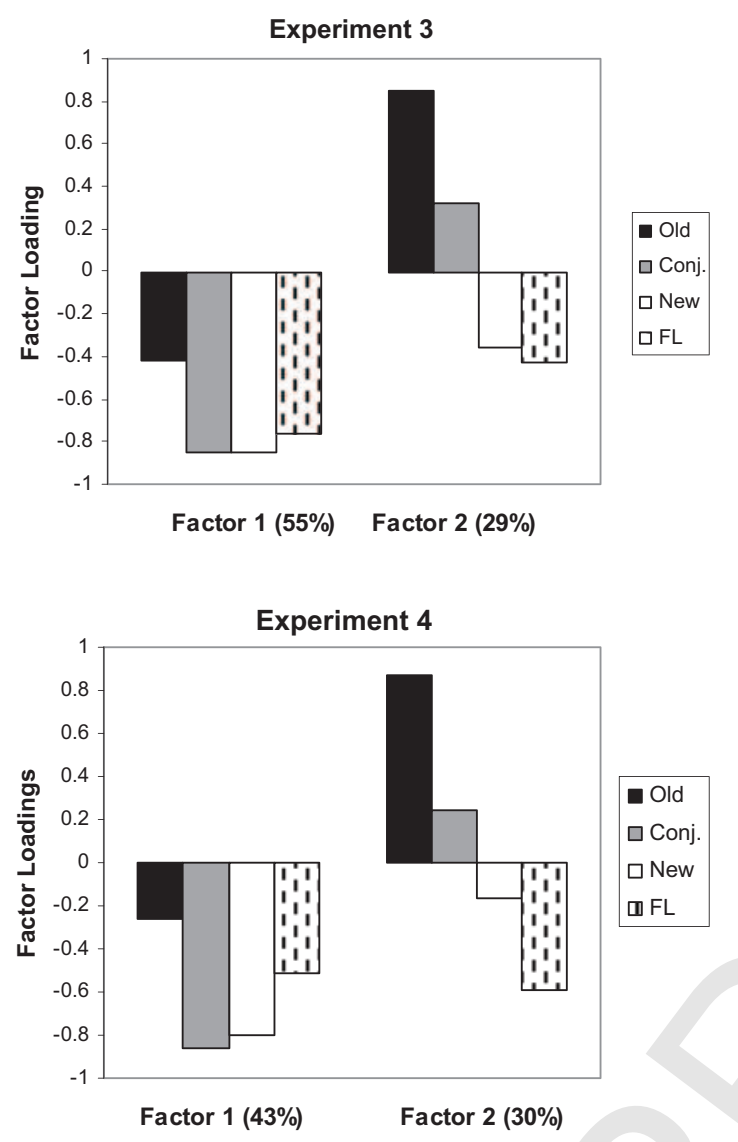

(B)
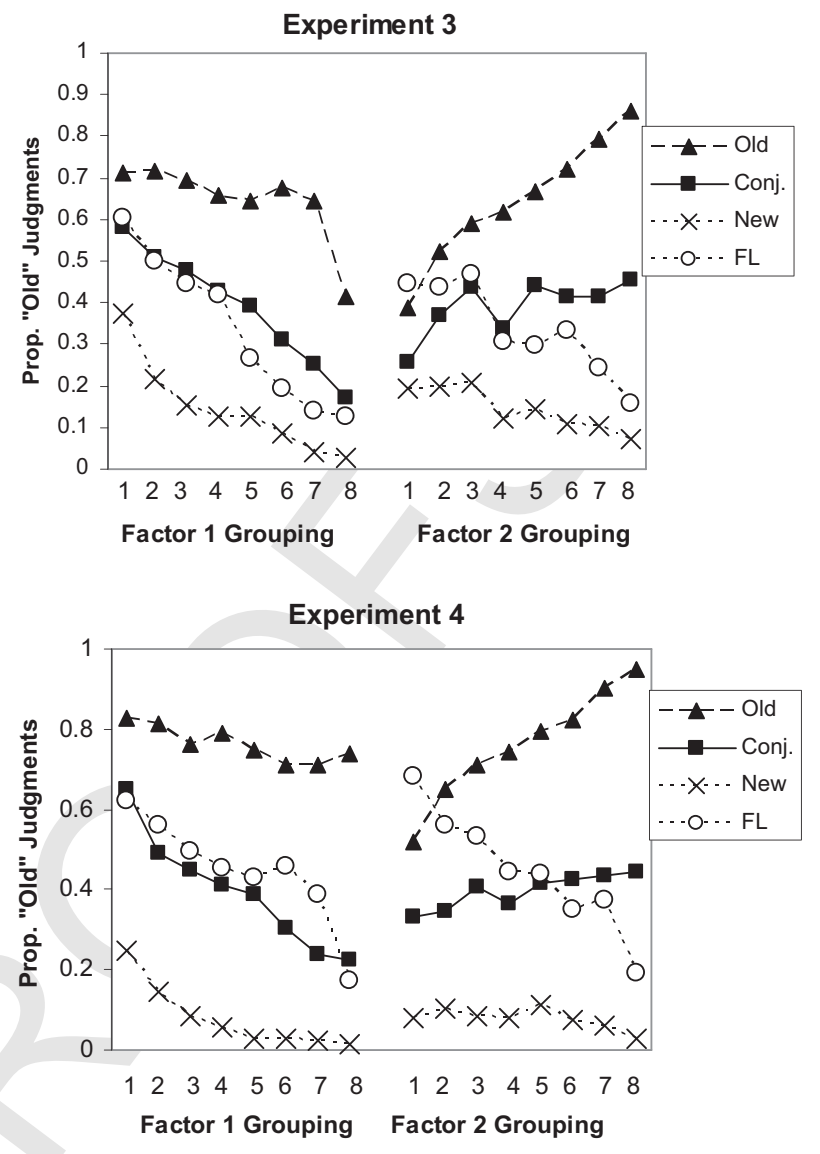

Figure 12. A: Loadings for hits to old faces and false alarms to conjunctions (Conj.), new faces, and familiarized lures (FL) on Factors 1 and 2 emerging from the principal-components analyses of Experiment 3 (top) and Experiment 4 (bottom). B: Hit rates for old faces and false-alarm rates for conjunctions, new faces, and familiarized lures in eight subsets of participants in Experiment 3 (top) and in Experiment 4 (bottom), grouped by Factor 1 scores (left) and Factor 2 scores (right).

tions, the recognition test included study-list faces and FLs, along with conjunctions and entirely new faces.

Our thinking was that, in the $1 \times-\mathrm{FL}$ condition, the old/FL discrimination might be based to some extent on familiarity differences, as the FL items were shown only once before the test but the old items were shown three times. In the $3 \times$-FL condition, however, familiarity should be approximately the same for FL and old items (both presented three times). Hence, we assumed that the old/FL discrimination in the $3 \times$-FL condition would be based primarily on context retrieval. To test this assumption, we planned to examine receiver operating characteristics (ROCs) relating hits and false alarms across five levels of criterion in each experimental condition. Although ROCs usually have a curvilinear, convex shape, Yonelinas $(1997,1999)$ found linear ROCs when recognition judgments were based on context retrieval. On the basis of this work, we expected linear ROCs for hits versus FL false alarms in the $3 \times$-FL condition, though not necessarily in the $1 \times-$ FL condition. The key question was whether Factor 2 would show a robust correlation with old/FL discrimination not only in the $1 \times$
-FL condition (where familiarity might support discrimination to some degree) but also in the $3 \times$-FL condition (where its contribution should be minimal). A strong correlation in the $3 \times$-FL condition would indicate that Factor 2 is linked to context retrieval.

\section{Method}

The 144 participants in Experiment 4 were recruited as in Experiment 3. The design and procedure were similar as well, except that (a) the study and test lists were shorter (64 and 128 faces, respectively, as opposed to 120 and 240, respectively, in Experiment 3) and (b) the study list (plus one filler at the start and one at the end) was preceded by a familiarization set of 32 faces. The familiarization set was shown one time to half of the participants $(1 \times-F L$ condition) and three times (in different randomized orders) to the remainder $(3 \times-\mathrm{FL}$ condition). The familiarization task in the $1 \times-\mathrm{FL}$ condition was to rate faces for trustworthiness, and the task in the $3 \times$-FL condition was to rate the faces for trustworthiness during the first presentation, attrac- 
tiveness during the second presentation, and intelligence during the third presentation. The study list was presented three times to all participants (in a different sequential order each time), and the task was simply to study the faces (as in Experiment 3). The subsequent test included 32 faces of each of four types (old, conjunction, new, and FL), presented in a randomized order. Participants were asked to judge whether each test face was or was not from the study list, according to a six-point confidence scale.

\section{Results and Discussion}

Hit and false-alarm rates. The lower panel of Figure 11 displays the hit rates for old faces and the false-alarm rates for conjunction, new, and FL faces in the $1 \times-\mathrm{FL}$ and $3 \times-\mathrm{FL}$ conditions. As in Experiment 3, hits were generally more frequent than false alarms, but false alarms were more frequent in response to conjunctions and FLs than in response to entirely new faces. The findings were similar in the $1 \times-\mathrm{FL}$ and $3 \times$-FL conditions, but note that the $3 \times$-FL condition produced slightly lower hit rates for old faces and slightly higher false-alarm rates for FLs. The pattern suggests that, when the familiarity difference between old faces and FLs was minimized, discrimination between the two item types was slightly impaired. To test this observation, we computed $d^{\prime}$ scores for discrimination between old faces and each of the other item types. Old/FL discrimination was reliably higher in the $1 \times$-FL condition than in the $3 \times-F L$ condition (mean $d^{\prime} s=1.24$ and $0.69, S D \mathrm{~s}=0.90$ and 1.13 , respectively), $F(1$, $42)=10.3, M S E=1.05, p<.01$. By contrast, old/conjunction discrimination and old/new discrimination were not reliably affected by experimental condition.

Converging evidence that the familiarity difference between old and FL faces was minimized in the $3 \times$-FL condition comes from a ROC analysis. Using each participant's recognition-confidence ratings, we computed hit rates for old faces, false-alarm rates for conjunctions, and false-alarm rates for FLs at each of five criterion levels; we used the means across participants to plot ROC curves for old/conjunction discrimination and for old/FL discrimination in the $1 \times$-FL and $3 \times$-FL conditions. As shown in Figure 13, old/FL discrimination in the $3 \times$-FL condition was associated with a linear ROC; this association suggests that such discrimination was based largely on recollection (context retrieval). By contrast, ROCs for old/FL discrimination in the $1 \times$-FL condition and for old/conjunction discrimination in both $1 \times-\mathrm{FL}$ and $3 \times$ -FL conditions were curvilinear; this indicates that familiarity played at least a partial role.

To quantitatively evaluate these findings, we compared the fit of the four group-level ROCs for two different two-parameter models, one assuming that familiarity is the sole basis of performance and a second assuming that only recollection is involved. The familiarity-only model estimated parameters for $d^{\prime}$ and the ratio of the target and lure distribution variance, whereas the recollectiononly model estimated parameters for recollection-based acceptance of targets and recollection-based rejection of lures (see Yonelinas \& Parks, 2006, for the Excel-based model-fitting program). The familiarity-only model produced a better fit than did the recollection-only model for old/FL discrimination in the $1 \times$ -FL condition (sum of squared errors $[S S E]=.00004$ vs. .002) and for old/conjunction discrimination in the $1 \times$-FL condition $(S S E=.00007$ vs. .002$)$ and in the $3 \times-$ FL condition $(S S E=$

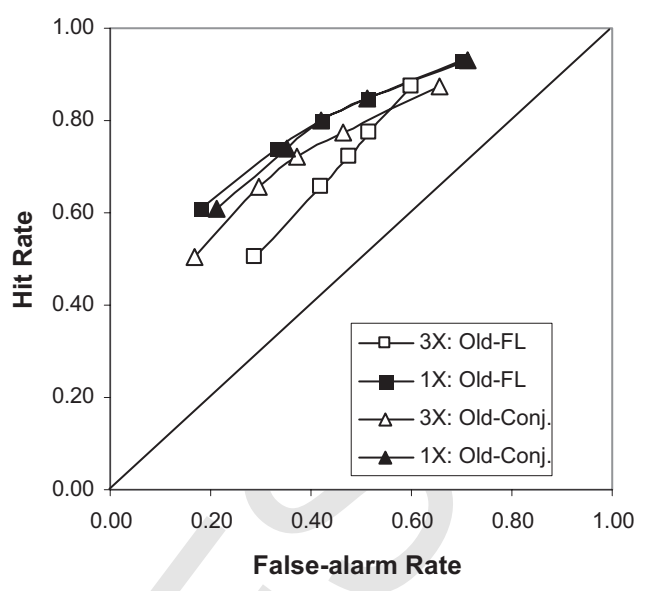

Figure 13. Receiver operating characteristics (ROCs) for discrimination between old faces and familiarized lures (Old-FL) and between old faces and conjunctions (Old-Conj.) for participants who viewed the familiarization set one time $(1 \times-\mathrm{FL})$ or three times $(3 \times-\mathrm{FL})$ in Experiment 4 . The ROC for Old-FL discrimination approximates a straight line in the $3 \times$-FL condition.

.0001 vs. .002). By contrast, the recollection-only model produced a better fit for old/FL discrimination in the $3 \times$-FL condition $(S S E=.00003$ vs. .006). This suggests that recollection was a major basis of performance in that condition.

We also fitted the data to a three-parameter dual-process model that provided estimates of recollection-based acceptance, recollection-based rejection, and familiarity $\left(d^{\prime}\right)$. The fits to the three-parameter model were good $(S S E=.00001$ and.000005 for old/FL discrimination in the $3 \times-$ FL and $1 \times-$ FL conditions and SSE $=.003$ and .00008 for old/conjunction discrimination in the $3 \times \mathrm{F}$ and $1 \times \mathrm{F}$ conditions), and the parameter estimations were in line with expectations. For old-FL discrimination in the $1 \times$-FL condition and old-conjunction discrimination in the $3 \times$-FL and $1 \times$-FL conditions, the $d^{\prime}$ estimates were $0.79,0.65$, and 0.91 , respectively. The recollection-acceptance estimates were 0.29 , 0.22 , and 0.13 , respectively, and the recollection-rejection estimates were essentially null ( 0.005 or less). By contrast, for old-FL discrimination in the $3 \times$-FL condition, the familiarity estimate was near 0 (and numerically negative, $d^{\prime}=-0.10$ ), whereas both recollection estimates were substantial $(0.21$ for recollectionacceptance and 0.30 for recollection-rejection). Because averaging artifacts can affect group-level ROCs, we fitted the dual-process model to the old-FL data from each participant who showed at least a 4-point old-FL ROC and above-chance old-FL discrimination $(n \mathrm{~s}=42$ and 54 for the $3 \times-\mathrm{FL}$ and $1 \times-\mathrm{FL}$ conditions, respectively). The estimates of $d^{\prime}$, recollection-acceptance, and recollection-rejection averaged $0.31,0.36$, and 0.14 , respectively, in the $1 \times$-FL condition and $-.18,26$, and.36, respectively, in the $3 \times$-FL condition (the SSE values averaged .002 in both cases). All parameters were reliably greater than $0(p \mathrm{~s}<.000001$ by $t$ test) except for the $d^{\prime}$ parameter in the $3 \times$-FL condition, which was numerically negative $(M=-0.18)$ and not different from 0 , $t(41)=1.28, p>.20$.

$P C A$. The lower half of Table 5 displays the intercorrelations among the hit-rate and false-alarm-rate measures along with the 
factor loadings of each measure on the first two components emerging from the PCA (eigenvalues $=1.71$ and 1.19 , respectively). The lower-left quadrant of Figure 12 displays the factor loadings, and the lower-right quadrant displays hit and false-alarm rates for eight subgroups of 18 participants each, sorted by their Factor 1 and 2 scores. The pattern is similar to that of Experiment 3 , although floor effects appear to have weakened the relation between Factor 2 scores and the conjunction effect. Again, we quantified this relation by computing the correlation between individual participants' Factor 2 scores and the conjunction/new difference in individual participants' false-alarm rates. The correlation was reliable, $r(142)=.40, p<.01$, albeit weaker than in Experiment 3 (where $r=.72$ ).

The most important finding here is that Experiment 4 replicated the negative relation between Factor 2 scores and FL false alarms and did so in the $3 \times$-FL condition as well as in the $1 \times-F L$ condition. As shown in Figure 14, separate PCAs of the $1 \times$-FL condition and the $3 \times$-FL condition $(n=72$ in each case) produced highly similar two-component outcomes (eigenvalues of 1.94 and 1.23 for Factors 1 and 2, respectively, in the $1 \times$-FL condition and of 1.60 and 1.10 , respectively, in the $3 \times-\mathrm{FL}$ condition). Both PCAs produced the crisscrossed relation between conjunction false alarms and FL false alarms across the Factor 2 groups (see right side of upper-right and lower-right graphs in Figure 14). Further, in both conditions there was a strong correlation between Factor 2 scores and old/FL discrimination. Across the 72 participants in each condition, the correlation of Factor 2 scores with the old/FL difference in "old" judgments was .90 in the $1 \times$-FL condition and .91 in the $3 \times$-FL condition. ${ }^{8}$

In summary, Experiment 4 replicated the two-component outcome obtained in prior experiments. The key new finding was that old/FL discrimination in the $3 \times$-FL condition showed a linear ROC that was well fit by a recollection-only model that constrained familiarity to be 0 as well as by a recollection-plusfamiliarity model that estimated familiarity to be close to 0 . In light of this finding, the strong correlations between Factor 2 scores and old/FL discrimination in the $3 \times$-FL condition show that Factor 2 - our hit-rate (and conjunction-effect) component-is strongly linked to context retrieval.

\section{General Discussion}

\section{Two Components of Facial Recognition Memory}

The present research provides strong evidence that face recognition relies on two distinct processing components, one that is related to false recognition and another that is linked to correct recognition as well as to a false memory phenomenon known as the conjunction effect. Experiments 1 through 4 and the Searcy et al. (1999) study differed from each other in several respects, including age range of participants, stimulus materials, list length, and experimental design. Yet, PCAs of all five data sets consistently returned a two-component outcome, with the two components accounting for at least $58 \%$ of the variance in the recognition performance.

The first component (Factor 1) accounted for 36\% to 55\% of the variance across the data sets, and it was strongly related to falsealarm errors in response to conjunctions as well as new faces. Factor 1 loadings for these two lure types were virtually identical in our reanalysis of the Searcy et al. (1999) data ( -.79 and -.80 , respectively), in Experiment 1 ( -.85 and -.86 , respectively), in Experiment 3 ( -.85 and -.85 , respectively), and in Experiment 4 $(-.86$ and -.80 , respectively). Perhaps most strikingly, Factor 1 loadings in Experiment 2 were $-.75,-.77$, and -.77 for new faces, conjunctions in the one-presentation $(1 \times$-conjunction) condition, and conjunctions in the eight-presentations $(8 \times-$ conjunction) condition, respectively. This invariance in loadings occurred despite large differences in average false-alarm rates among the three item types $(M \mathrm{~s}=0.18,0.35$, and 0.46 , respectively; see Table 3). In Experiments 3 and 4, we included FLs, which were falsely recognized approximately as often as were conjunctions. Factor 1 loadings for FL false alarms were -.76 and -.52 in these two experiments. Although these loadings were somewhat weaker than the loadings for conjunctions and new faces, the data are largely in line with our conclusion that Factor 1 is linked to false alarms.

Underscoring this conclusion, Factor 1 loadings for hits were extremely low in the Searcy et al. (1999) data (.01), in Experiment 1 (.02), with eight-times presented $(8 \times$-old) items in Experiment 2 (.14), and in Experiment 4 (-.26). The loadings for hits were somewhat stronger for the poorly learned, once-presented $(1 \times$ old) items in Experiment $2(-.39)$ and in Experiment 3, in which the study list and recognition test were extremely long (-.42). Presumably, when learning is low and/or forgetting is high, Factor 1 might degenerate into a pure criterion factor, as the moderate loadings for hits in the same direction as false alarms suggest.

The second component emerging from the PCAs was also substantial, accounting for $22 \%$ to $39 \%$ of the variance across the five data sets. Our suggestion that this component is related to hits was consistently supported: The loadings of hit rates on Factor 2 were .85 or higher except in the case of the poorly learned once-presented $(1 \times$-old) items in Experiment 2, for which the hit-rate loading was 47.

Factor 2 was related not only to hits but also to conjunction false alarms and new-face false alarms, though in opposite directions. Although these false-alarm loadings were moderate in size, their sign reversals for the two lure types produced an interesting effect: Factor 2 scores were consistently related to the conjunction effect. The Factor 2/conjunction-effect correlation was .65 in the Searcy et al. (1999) data and .64, .49, .72, and .40 in Experiments 1 through 4 , respectively. Though not as strong as the hit-rate loadings, these correlations were substantial and statistically reliable. Further, the relatively low correlation of .40 in Experiment 4 can be attributed to a floor effect on new-face false alarms.

\section{The Rapcsak et al. (1999) Model and Alternative Conceptions of the Two Components}

Our PCAs supported the existence of two separable components that we have referred to, for convenience, as a false-alarm-rate component and a hit-rate component. It is critical, however, to move beyond mere labeling to characterize these components in terms of theory. We began this research with the hypothesis that the two components emerging from our PCAs reflected the con-

\footnotetext{
${ }^{8}$ The corresponding correlations of Factor 2 scores with $d$ 's for old/FL discrimination were .92 and .87 , respectively.
} 
(A)

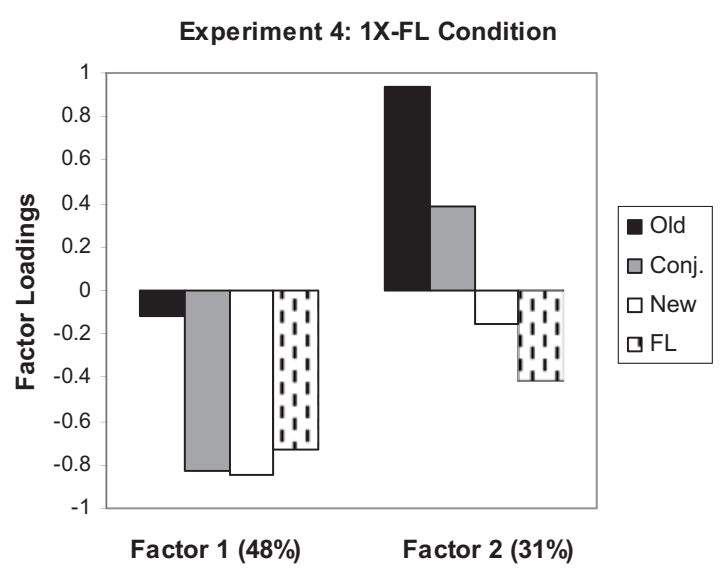

Experiment 4: 3X-FL Condition

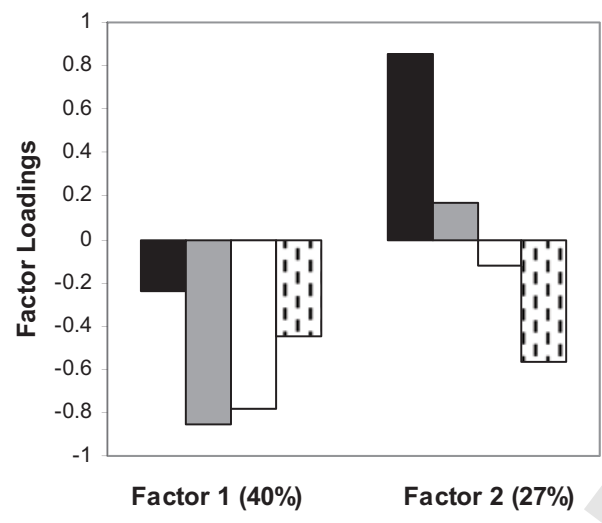

(B)
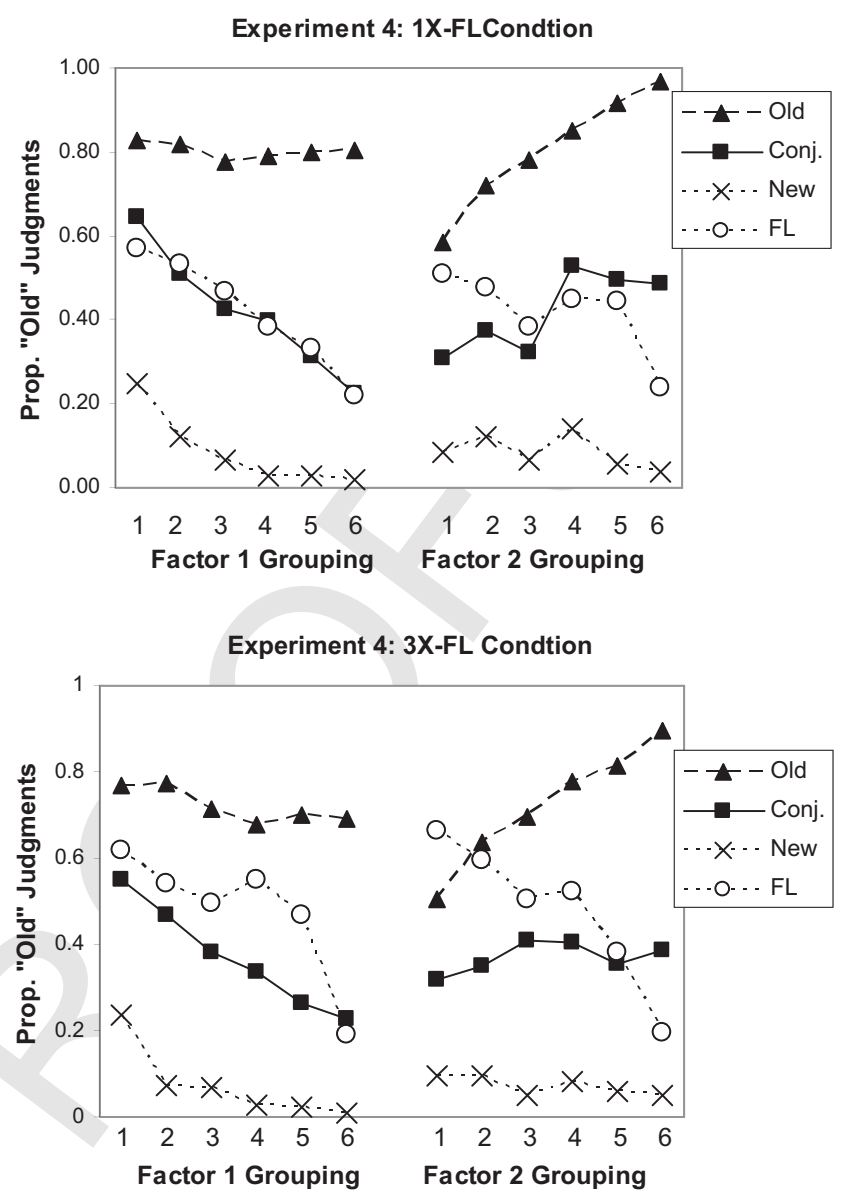

Figure 14. A: Loadings for hits to old faces and false alarms to conjunctions (Conj.), new faces, and familiarized lures (FL) on Factors 1 and 2 emerging from separate principal-components analyses of the one-time familiarization $(1 \times-\mathrm{FL}$, top $)$ and three-times familiarization $(3 \times-\mathrm{FL}$, bottom $)$ conditions of Experiment 4 . B: Hit rates for old faces and false-alarm rates for conjunctions, new faces, and familiarized lures in six subsets of participants in the $1 \times$-FL (top) and $3 \times$-FL (bottom) conditions of Experiment 4, grouped by Factor 1 scores (left) and Factor 2 scores (right).

tributions of the frontal and occipitotemporal components of Rapcsak et al.'s (1999) neuropsychological model of face recognition memory (see Figure 1). The hypothesis has fared well, as the PCA findings suggest a component that-like Rapcsak et al.'s (1999) "frontal executive" component-serves to reduce false alarms. These findings also suggest another component that-like Rapcsak et al.'s occipitotemporal "face recognition module"-works to increase hits. We find it impressive that our first component was related to false-alarm errors with three different types of lure (conjunctions, FLs, and new faces). Because it is unlikely that all three types of lure were rejected through the same process, this outcome suggests that the component reflects control of falsealarm errors through a number of different processes, as does the frontal executive component in the Rapcsak et al. (1999) model. That Factor 1 approximated a criterion factor under conditions of low learning and high interference is also consistent with the model. According to the model, the setting of recognition criteria is one way that the frontal executive component works to limit false alarms. In conditions of low learning and/or high interference, it may be the most important way.

The Rapcsak et al. (1999) model also captures our finding that the hit-rate component was related not only to hits but also to conjunction false alarms and FL false alarms in opposite directions. A core claim of the model is that the occipitotemporal component - the face recognition module - can trigger bottom-up retrieval of contextual information. Such information is likely to be helpful in accepting old test items and also in rejecting FL items (which were encountered outside of the study-list context) but not in rejecting conjunctions (whose parents were encountered within the study-list context). Thus, a face recognition component that triggers bottom-up retrieval of contextual information provides an unforced explanation of why Factor 2 showed a strong positive 
relation to hits, a negative relation to FL false alarms, and a positive relation to conjunction false alarms.

Three alternative characterizations of our two PCA components were considered in the course of this research. One characterization was that these two components reflect discrimination and criterion, as these concepts are defined in signal detection theory. However, contrary to this view, Experiment 1 revealed that each component was related to both aspects of performance. Another characterization was that one of our components reflected configural processing whereas the other reflected featural processing. On the basis of much evidence that inversion impairs configural processing more than featural processing (for a review, see Bartlett et al., 2003), this hypothesis predicts that one of our components should be weakened by inversion more than the other. However, contrary to this hypothesis, we found that neither component was exclusively sensitive to facial orientation and that both components emerged from recognition of inverted faces. Still a third characterization that we considered was that our two PCA components reflected the familiarity-recollection distinction in dualprocess theories of recognition memory (Jacoby, 1991, 1999), with Factor 2 reflecting context-free familiarity and Factor 1 reflecting recollection (i.e., the use of recollection to control false alarms). The hypothesis predicts that Factor 2 should show strong and same-direction loadings for (a) correct recognitions of study-list faces (hits) and (b) false recognitions of FLs. Contrary to the prediction, Factor 2 produced strong and reversed-sign loadings for hits and FL false alarms, and Factor 2 scores were strongly correlated with measures of old/FL discrimination $(r \mathrm{~s} \approx 90)$, even in conditions that minimized the contribution of familiarity to such discrimination (i.e., in the $3 \times$-FL condition of Experiment 4). We did not directly test the notion that Factor 1 is a recollectionrejection component. However, recollection-rejection of facial conjunctions appears to be minimal, even in conditions designed to maximize its occurrence (Jones \& Bartlett, 2009).

Apart from the hypotheses that we initially considered, several additional conceptions might be applied to our data and deserve consideration. One of these is fuzzy trace theory (Brainerd \& Reyna, 2005), an account that distinguishes verbatim representations of perceptual information from gist representations of meaning. Brainerd and Reyna (2005) proposed that gist representations for faces contain categorical information pertaining to race, gender, and general body build. They did not specify the nature of "verbatim" representations of faces, but presumably these contain highly detailed information about facial features and configuration, as well as item-specific episodic information. If we accept this characterization, fuzzy trace theory nicely handles our finding that Factor 1 (the verbatim component?) was linked to discrimination between old faces and three different types of lure (conjunctions, new faces, and FLs). The theory is also in line with the finding that Factor 2 (the gist component?) was positively related to the false recognition of conjunction items because, presumably, conjunctions match well with gist information. Despite its strengths, however, the theory requires elaboration to explain why Factor 2 was negatively related to false recognition of FLs. In addition, a key claim of fuzzy trace theory is that fuzzy and verbatim traces work together to support correct recognitions. Thus, without elaboration, the theory does not explain why only one of our components showed strong loadings for hits.
Another relevant theoretical conception distinguishes postretrieval monitoring from the initial process-whatever its nature-that underlies the retrieval or activation of memories in response to a cue (Moscovitch, 1994Odegard \& Lampinen, 2006; Roediger \& McDermott, 2000; Schacter, 2001). Post-retrieval monitoring is viewed as important for reducing false alarms through assessments of (a) the distinctiveness of the retrieved information (e.g., Dodson \& Schacter, 2002), (b) information specifying its context or source (e.g., Roediger \& McDermott, 2000), or (c) the goodness or completeness of match between a test cue and a memory trace (Reder, Wible, \& Martin, 1986). The idea is similar to the Rapcsak et al. (1999) conception that a frontal executive component performs such monitoring (along with other functions). Yet, like the dual-process and fuzzy-trace hypotheses, it does not explain why Factor 2 showed opposite-direction loadings for false recognitions of conjunctions and FLs. Our application of the Rapcsak et al. (1999) model is preferred in this regard, as it provides a simple, unforced explanation of why the hit-rate component should be positively correlated with false recognitions of conjunctions and yet negatively correlated with false recognitions of FLs.

A final hypothesis we consider is the inhibitory deficit theory of Hasher, Zacks, and their associates (Hasher \& Zacks, 1988; Lustig, Hasher, \& Zacks, 2007). These researchers argued that inhibition reflects "the ability to limit activation to information most relevant to one's goals" (Lustig et al., 2007, p. 146). They distinguished inhibition from activation and argued that inhibition is the strongest source of individual differences in cognitive tasks.

From the perspective of inhibitory deficit theory, it is tempting to link our false-alarm-rate component with inhibition and our hit-rate (and conjunction-effect) component with activation. If these connections are made, the theory nicely handles the finding that hits showed a strong positive loading on only one of our components. Second, because inhibition is a larger source of individual differences than is activation, the theory predicts that Factor 1-our false-alarm-rate component-not only should exist but should also account for more variance than does Factor 2 (our hit-rate component). None of the other aforementioned theories make this prediction, and yet it was supported in all five data sets considered in this report. These strengths notwithstanding, the theory must be elaborated if it is to explain why the hit-rate component was positively correlated with false recognitions of conjunctions and yet negatively correlated with false recognitions of FLs, a finding neatly captured by our application of the Rapcsak et al. (1999) model.

In arguing for the Rapcsak et al. (1999) model as a framework for our findings, we are not claiming that it is superior in other respects to the various alternatives we have considered. Our point is more narrow: The Rapcsak et al. model appears to handle individual differences in recognition of faces better than do the alternatives. This is no mean achievement, but it is not everything. Consider our conclusion that neither of our factors reflects the process of familiarity posited by dual-process theories. Although our data strongly support this conclusion, they do not rule out a familiarity process, and in fact the Rapcsak et al. model includes a familiarity component (resemblance). A reasonable idea, quite consistent with our data, is that familiarity is real and important but that it does differ much across healthy individuals. 


\section{Generality of the Two Components}

An issue attending face processing research is whether the findings and relevant models are specific to faces. In the Rapcsak et al. (1999) model, the characterization of the occipitotemporal component as a "face recognition module" implies it is specific to faces (see Fodor, 1983, for discussion of modules). Further, McKone and Peh (2006) have argued that general memory theories (which they call "memory-only" theories) do not work well with faces, as, for example, these conceptions do not explain why facial inversion impairs face recognition accuracy. In support of their argument, McKone and Peh reported two studies using the conjunction paradigm that showed that inversion impaired discrimination between old faces and conjunctions, a finding we have also reported (Bartlett et al., 2003) and replicated here in Experiments 1 and 2.

Although facial inversion has effects that are not handled by general memory theories, it is important to consider certain qualitative similarities in recognition memory for upright and inverted faces. Inversion reduced discrimination between old faces and conjunctions, but it left the basic conjunction effect- the conjunction/new difference in false-alarm errors-largely unaffected (both here and in McKone \& Peh, 2006). Moreover, the effects of presentation frequency on conjunction false alarms were impressively similar for upright and inverted faces in Experiment 2 (see Table 3). Finally, our success in replicating the dual-component outcome of our PCAs with inverted faces (see Figure 10) is another instance of a qualitative similarity, and it suggests that our dual components might generalize across stimulus domains.

Further evidence that the dual-component outcome has impressive generality comes from an experiment by Rubin, Van Petten, Glisky, and Newberg (1999). Their young and senior participants received a study list of disyllabic words followed by a test including old and new words, together with conjunctions (each combining the first syllable of one study-list word with the second syllable of another) and "syllable" lures (each combining one old syllable with one new syllable). The senior participants also took two neuropsychological test batteries, one for frontal function and the other for temporal lobe function. The upper graphs of Figure 15 compare the high and low performers on the frontal battery (upperleft graph) and the high and low performers on the temporal lobe battery (upper-right graph). Note that the frontal scores were related primarily to false-alarm errors whereas, by contrast, the temporal-lobe scores were related to hits as well as to the conjunction/new difference in false alarms (false-alarm rates for syllable lures fell between those for conjunctions and for entirely new items). For comparison, we have included data from our reanalysis of the Searcy et al. (1999) face recognition study in the lower graphs of Figure 15. These graphs compare participants with above- and below-average Factor 1 scores (lower-left graph) and above- and below-average Factor 2 scores (lower-right graph). These are the same data shown in Figure 4, except that we have divided the participants into two groups instead of six. Despite a difference between studies in the overall level of conjunction false alarms, Factor 1 is related to face recognition in the same way that frontal-test performance was related to word recognition performance in Rubin et al. (1999). Similarly, Factor 2 is related to face recognition in the same way that performace on the temporal-lobe test was related to word recognition.
A second relevant study by Stark and Squire (2003) compared patients who had suffered hippocampal damage with healthy controls in the conjunction paradigm. All participants performed five recognition memory tasks with five different types of stimuli: disyllabic words, compound words, word pairs, object pairs, and face-house pairs. In each task, a study list was followed by a recognition test that included old items, conjunctions, and entirely new items (it also included feature lures, each composed of one old and one new part). As shown in Figure 16, the controls greatly F16 exceeded the patients in hit rates to old items, and they also showed a stronger conjunction/new difference in false-alarm errors. ${ }^{9}$ The pattern is similar to the one we obtained when we Fn9 divided the participants in Experiment 2 (or any other of our experiments) into high versus low Factor 2 groups (see lower-right graph in Figure 16). We believe our analyses of these previously reported studies support the contention that the dual-component outcome of our PCAs reflects general frontal and medial temporal lobe (MTL) components that are not restricted to faces.

In light of qualitative similarities between recognition of upright faces and recognition of other stimulus types, it would appear ill advised to develop theories of face recognition without consideration of how general memory processes might be involved. Rather, we should attempt to identify and characterize those components of face recognition memory that are domain specific (used only with faces) or domain-related (relatively more important with faces than with other stimuli) and to explore how these components interact with general memory processes. A proposed elaboration of the Rapcsak et al. (1999) model (see Figure 17) takes a $\mathbf{F 1 7}$ step in this direction.

\section{A Proposed Modification of the Rapcsak et al. Model}

Our modification of the Rapcsak et al. (1999) model derives from recent evidence that recognition memory for upright faces is based on both configural and featural information (though the former appears to be more important in many situations; see Bartlett \& Searcy, 1993; Bartlett et al., 2003; Cabeza \& Kato, 2000; McKone et al., 2007; Moscovitch, Winocur, \& Behrmann, 1997; Sergent, 1984) and that configural face processing involves (at least) two occipitotemporal regions (Rossion et al., 2003; Schiltz \& Rossion, 2006) that are distinguishable from the MTL structures that are known to be critical for recognition memory with a range of different stimuli and that collectively support both resemblance (familiarity) and recollection (Eichenbaum, Yonelinas, \& Ranganath, 2007; Mayes, Montaldi, \& Migo, 2007; Squire, Stark, \& Clark, 2004). Controversy swirls as to whether the processing of upright faces is performed by a prewired module or is linked to expertise and also as to whether resemblance and recollection involve separate or overlapping MTL structures. Nonetheless, there is sufficient consensus on these points for us to consider updating the Rapcsak et al. (1999) model, as shown in Figure 17.

The core idea of this modified model is that face recognition involves (a) part-based processing that can support recognition of upright faces as well as objects and inverted faces and (b) holistic/ configural processing that can support recognition of upright faces much more than recognition of objects and inverted faces (see

\footnotetext{
${ }^{9}$ The hit rates in the Stark and Squire (2003) experiments were estimated from false-alarm rates and $d^{\prime}$ values reported in the article.
} 


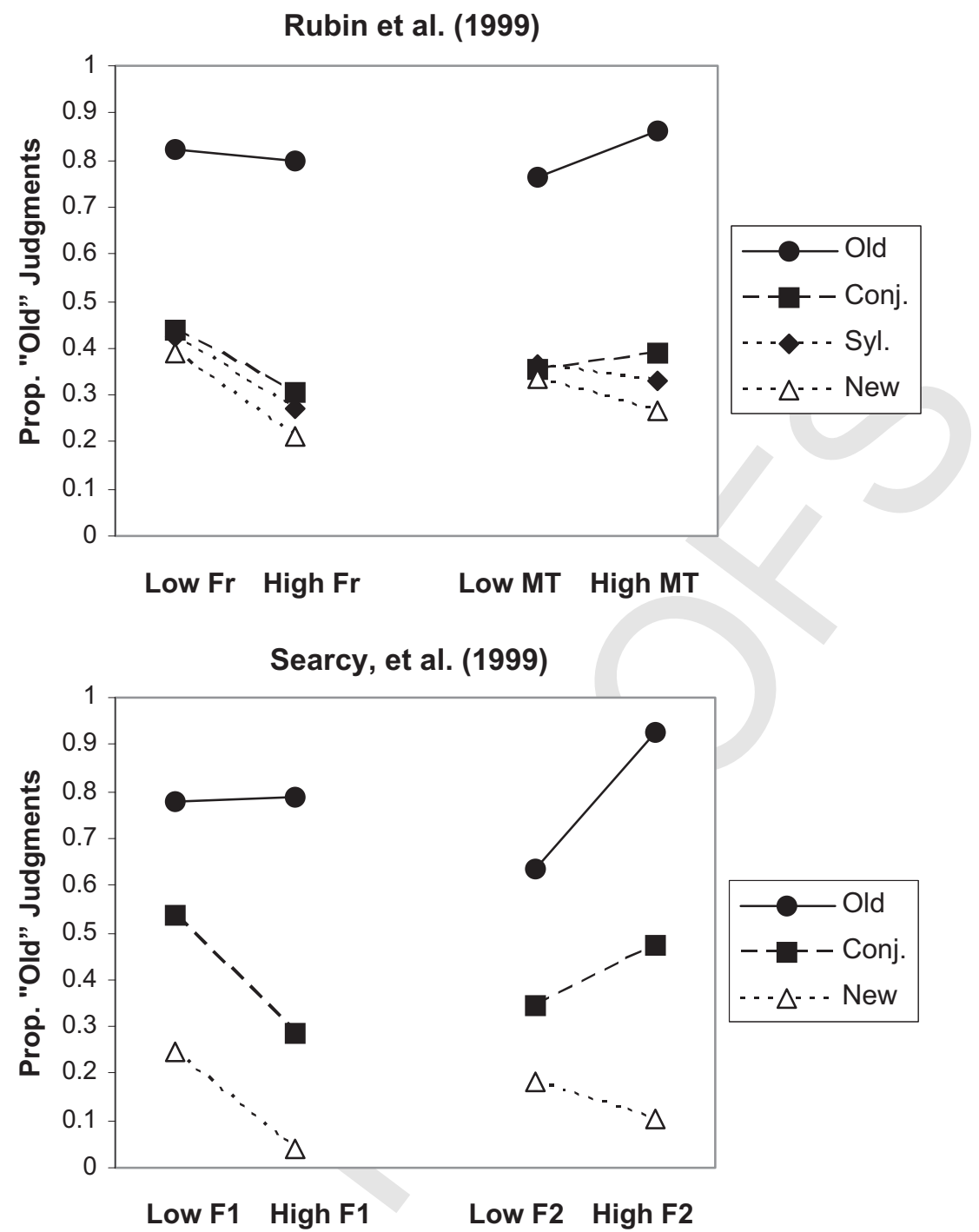

Figure 15. The upper graph shows hit rates for old items and false-alarm rates for conjunction (Conj.), syllable (Syl.), and new items for senior participants scoring high versus low on frontal (FR; left) and medial-temporal (MT; right) test batteries in the Rubin et al. (1999) verbal recognition memory study. The lower graph shows hit rates for old faces and false-alarm rates for conjunction and new faces for participants who had high versus low Factor 1 (left) and Factor 2 (right) scores in the Searcy et al. (1999) study.

Bartlett et al., 2003; McKone, Martini, \& Nakayama, 2003; Tanaka \& Farah, 2003). In a recognition test, part-based processing signals part-based resemblance, which is considerably higher for conjunctions (as they are composed of old parts) than for new faces. By contrast, holistic/configural processing signals configural resemblance, and this is assumed to be generally much higher for old faces (as they are exact copies of study-list faces) than for conjunctions and new faces. Resemblance signals from both components are sent to the MTL memory component, which computes overall resemblance strength and can trigger bottom-up retrieval of contextual information. We assume that both resemblance strength and bottom-up retrieval of context are greater for old faces than for conjunctions (largely due to holistic/configural processing) and are greater for conjunctions than for new faces (largely due to partbased processing).

The output of the MTL memory component-resemblance signals and contextual information-is monitored by the frontal executive component (FEC). The FEC can operate either in an automatic, bottom-up mode or in a controlled, top-down mode. In the bottom-up mode (signified by the upward-pointing arrows in Figure 17), the FEC derives a value of "memory strength" as a cumulative function of both bottom-up inputs from MTL (the upward-pointing arrows to the FEC in Figure 17). In the top-down mode (see downward-pointing arrows), the FEC works to reduce false alarms by (a) checking the extent to which the resemblance signal reflects configural resemblance versus part-based resem- 
Expt. 1: Disyllabic Words

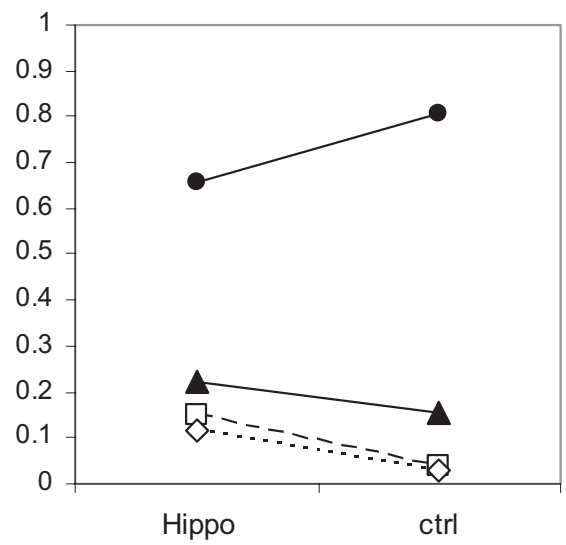

Expt. 2: Psuedo-Compound Words

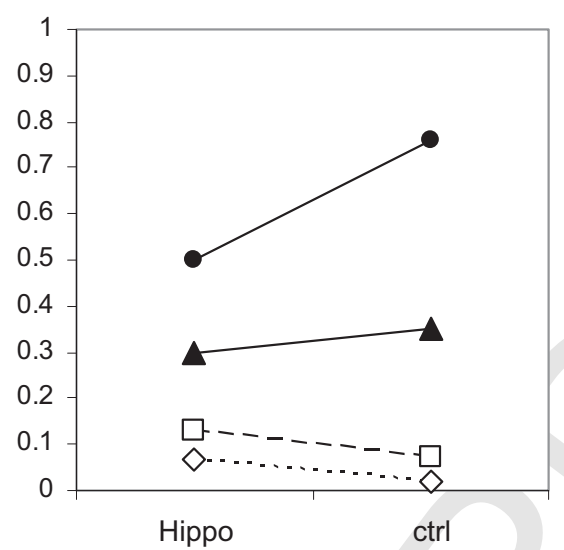

Expt. 3: Word Pairs

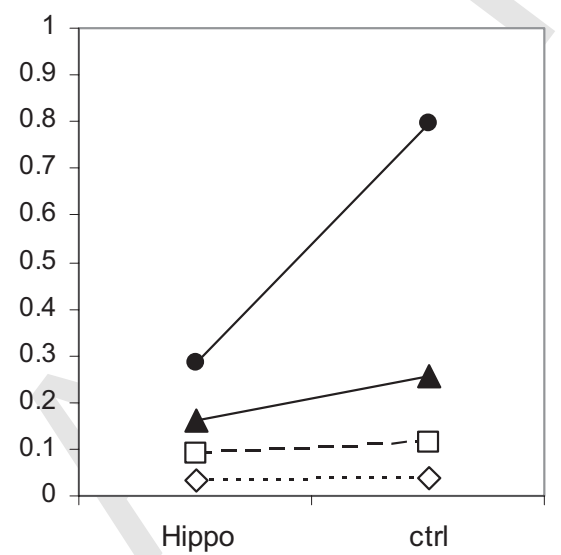

Expt. 4: Object Pairs

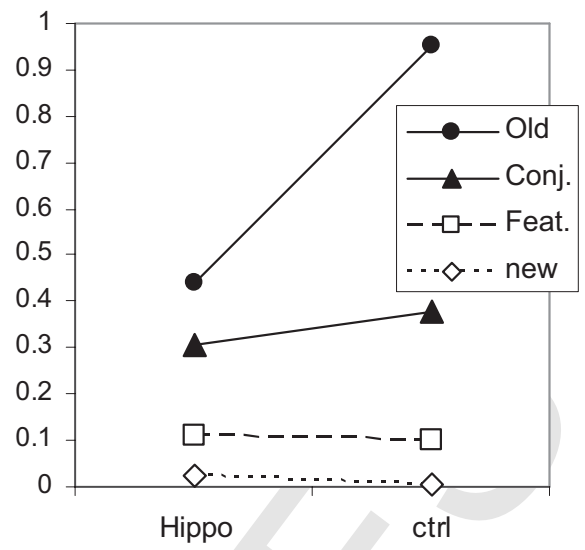

Expt. 5: House-Face Pairs

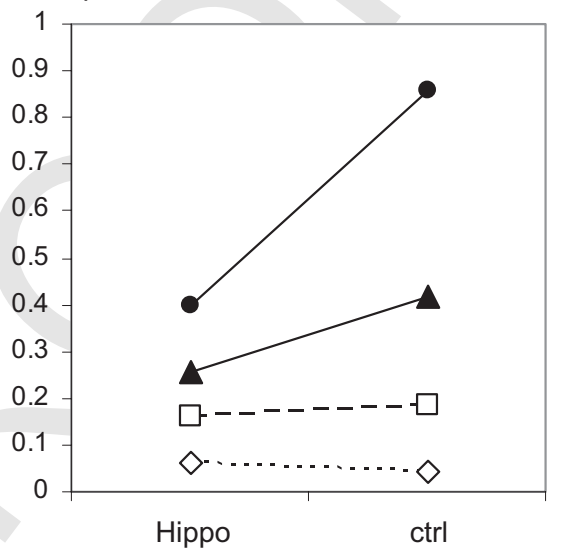

Bartlett et al., Expt. 1, Faces

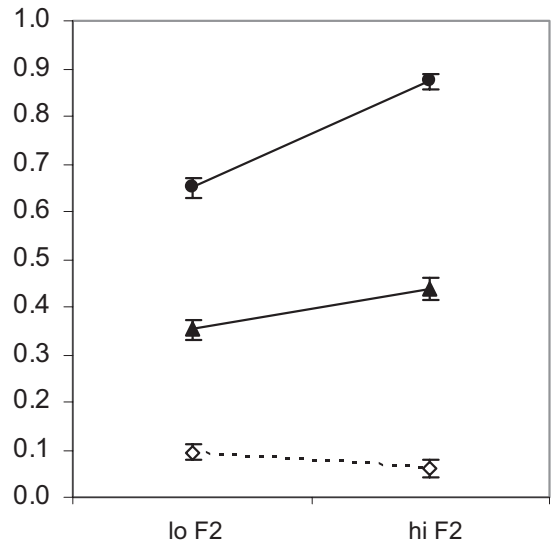

Figure 16. Hit rates for old items and false-alarm rates for old, conjunction (Conj.), feature (Feat.), and new items for hippocampally damaged (Hippo), and control (Ctrl) participants in the five experiments reported by Stark and Squire (2003), along with hit rates for old faces and false-alarm rates for conjunction and new faces for high- and low-Factor-2 participants in Experiment 1. Error bars depict standard errors of the means.

blance (see arrow A in Figure 17) and (b) examining contextual/ associative information for evidence that the various parts of a face were encountered together (i.e., in the same context; see arrow B in the figure).
We propose that the hit-rate (and conjunction-effect) component emerging from these studies reflects primarily the amount of contextual/associative information retrieved in the bottom-up mode (though it may reflect resemblance information to some 


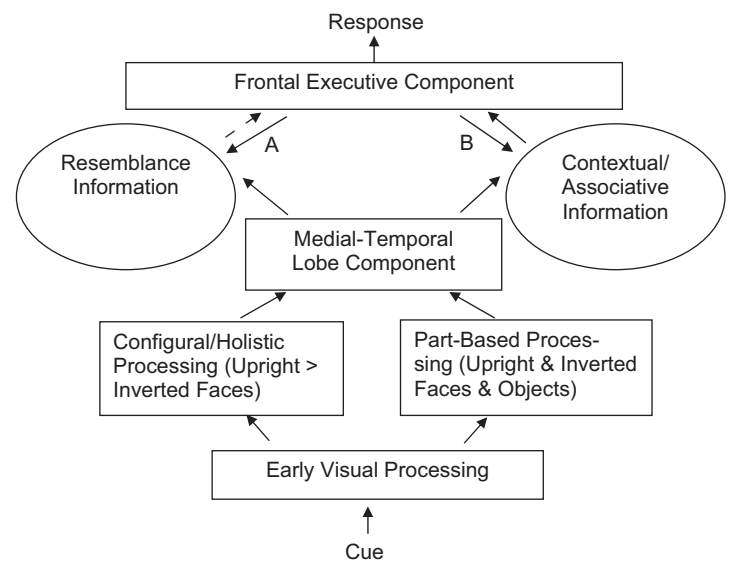

Figure 17. A modified model of face recognition, based on Rapcsak et al. (1999), with upward-pointing arrows representing bottom-up perceptual and retrieval processes and downward-pointing arrows representing controlled, top-down processes of (A) careful analysis of resemblance information, and (B) careful analysis of contextual/associative information (the latter includes biographic information in the case of well-known faces). The broken arrow from "resemblance information" to the frontal executive component reflects the assumption that resemblance information is weighed less heavily than contextual/associative information in bottom-up recognition.

extent). We further propose that the false-alarm-rate component reflects the extent of top-down analysis of resemblance information and contextual/associative information in making recognition judgments. Participants who are strongest on this second component follow a decision strategy whereby "old" judgments are reserved for faces that (a) produce strong configural resemblance relative to part-based resemblance and (b) evoke retrieval of contextual/associative information specifying that various parts of a face were studied close together in time. By this modified model, one effect of facial inversion is to reduce memory strength for old faces relative to that for conjunctions and new faces. This will cause inversion to reduce hit rates if participants are functioning in the bottom-up mode. A second effect is to disable the top-down configural-match test, which can aid lure rejection in the top-down mode. This will cause inversion to increase false alarms if participants are functioning in the top-down mode.

We believe that these assumptions may explain why inversion had its variable effects, decreasing hits (and decreasing Factor 2 scores) in the proximal condition of Experiment $2 b$ and increasing false alarms (and reducing Factor 1 scores) in the nonproximal condition of Experiment 2b, in Experiment 2a, and in Experiment 1. By this account, participants in the proximal condition relied primarily on the bottom-up mode, possibly because the top-down contextual/associative test was useless for rejecting conjunctions in that condition (in which conjunctions' parents had been studied close together).

\section{Independence of the Two Components}

After perusing our data, colleagues have questioned whether it is plausible that a MTL component and a FEC would be truly orthogonal, as our PCAs suggest. Is not it likely that two such components would interact with each other and therefore would be correlated? Our first response to this question is that two such components almost certainly interact, but this does not mean the corresponding individual differences are correlated. An analogy might be drawn to how a modern car's engine interacts with its automatic transmission. An increase or decrease in RPMs will cause a gear change, and, likewise, a change of gears can affect RPMs. However, this does not mean that there must a correlation between the engine horsepower and number of gears across a set of cars.

Our second response is based on a hierarchical factor analysis that we performed on the data from all five data sets. Hierarchical factor analysis (Wherry, 1984) derives oblique factors by identifying clusters of the stronger loadings emerging from a PCA and rotating the axes in the factor space to go through these clusters. It then computes the correlations among these oblique factors and the original, primary, factors. Finally, it derives a general factor by performing a PCA on the correlation matrix of oblique factors and primary factors. Hence, the technique provides a quantitative assessment of the orthogonality of the factors and also assesses the importance of any higher order factor that might explain variance not accounted for by the primary factors.

In the case of Experiment 4, the two oblique factors were essentially uncorrelated with each other $(r=-.06)$, and each was almost perfectly correlated $(r=.97)$ with one of the primary factors. Further, the general factor had only very weak loadings (.28 or less) on the dependent measures (hits to old items and false alarms to conjunction, new, and FL faces). In sum, the analysis did not identify oblique factors that differed from the primary (orthogonal) factors, and it failed to identify a general factor with substantial loadings on our dependent measures. Essentially the same outcome was obtained from the data of Searcy et al. (1999), and Experiments 1 and 2. In Experiment 3, there was some suggestion of oblique factors that were slightly correlated with each other $(r=$ .36) and that were less than perfectly correlated with the primary factors $(r \mathrm{~s}=.80)$. Further, the general factor had same-direction loadings of .45 to .66 on the four dependent measures. This is in accordance with our prior conclusion that, due to the lengthy study list and recognition test, Factor 1 approximated a criterion factor in that experiment. In general, the hierarchical factor analyses did not suggest any substantive modification of the conclusions we drew from the original PCAs.

\section{Broader Implications}

We close by discussing three implications of the dualcomponent view of face recognition supported in this article. A first implication is that a model emerging from neuropsychological studies of patients with brain injuries was supported by individual difference research. This implication is important, because many types of brain damage might produce deficits that are dissociable from each other but have no bearing on those processes that vary independently among healthy adults. The present data stand as evidence that components such as those in the Rapcsak et al. (1999) model vary independently among healthy adults, and research should examine why this is the case.

A second implication concerns an interesting commonality between the Rapcsak et al. (1999) model we have favored in this 
article and inhibitory deficit theory (e.g., Lustig et al., 2007). Both of these models propose a component of cognition that does not represent a single process but rather a set of correlated processes that serve a common function. The frontal executive system of Rapcsak et al. (1999) has the function of controlling false alarms through criterion setting, source monitoring, and strategic search (we have proposed configural-match testing as well). In the inhibitory deficit theory, inhibition works to limit activation through controlling the focus of attention, deleting irrelevant information from working memory, and suppressing inappropriate responses. The delineated processes are presumably dissociable, but nonetheless they are correlated with each other. Rapcsak et al. (1999) characterized their frontal component as a "system," and by some definitions, it qualifies as such. Tulving (1985), for example, characterized a system as "a set of correlated processes" (p. 386). By other definitions, it probably does not (Schacter, Wagner, \& Buckner, 2000). In any case, there appears to be a place in contemporary theory for constructs that pertain to sets of correlated processes that serve a common function, whether they qualify as systems or not (see also Friedman et al., in press).

A third implication is more practical than others and is more specific to the problem of face recognition: Dimensions of difference among healthy persons - such as those identified by our Factors 1 and 2-suggest targets for training and other interventions to improve face recognition. False recognitions of faces are a major problem in eyewitness testimony, and so it is important to examine whether our first component generalizes to the lineup task and, if so, to determine whether regimens might be devised to help low-Factor-1 eyewitnesses avoid false identifications. Indeed, our PCA results may aid in understanding the variable effects of sequential presentation found in the lineup task.

\section{References}

Anderson, N. D., \& Craik, F. I. M. (2000). Memory in the aging brain. In E. Tulving \& F. I. M. Craik (Eds.), The Oxford handbook of memory (pp. 411-422). New York: Oxford University Press.

Bartlett, J. C. (1993). Limits on losses in face recognition. In J. Cerella, J. Rybash, W. Hoyer, \& M. Commons (Eds.), Adult information processing: Limits on loss (pp. 351-379). New York: Academic Press.

Bartlett, J. C., \& Memon, A. (2007). Eyewitness memory in young and older adults. In R. C. L. Lindsay, D. F. Ross, J. D. Read, \& M. P. Toglia (Eds.), Handbook of eyewitness psychology: Vol. II. Memory for people (pp. 309-338). New York: Psychology Press.

Bartlett, J. C., \& Searcy, J. H. (1993). Inversion and configuration of faces. Cognitive Psychology, 25, 281-316.

Bartlett, J. C., Searcy, J. H., \& Abdi, H. (2003). What are the routes to face recognition? In M. A. Peterson \& G. Rhodes (Eds.), Perception of faces, objects, and scenes: Analytic and holistic processes (pp. 21-52). London: Oxford University Press.

Bors, D. A., \& MacLeod, C. M. (1996). Individual differences in memory. In E. L. Bjork \& R. A. Bjork (Eds.), Handbook of perception and cognition: Vol. 10. Memory (pp. 411-441). San Diego, CA: Academic Press.

Brainerd, C. J., \& Reyna, V. F. (2005). The science of false memory. New York: Oxford University Press.

Cabeza, R., \& Kato, T. (2000). Features are also important: Contributions of featural and configural processing to face recognition. Psychological Science, 11, 429-433.

Charman, S. D., \& Wells, G. L. (2007). Applied lineup theory. In R. C. L. Lindsay, D. F. Ross, J. D. Read, \& M. P. Toglia (Eds.), Handbook of eyewitness psychology: Vol. II. Memory for people (pp. 219-254). New York: Psychology Press.

Damasio, A. R., Tranel, D., \& Damasio, H. (1990). Face agnosia and the neural substrates of memory. Annual Review of Neuroscience, 13, 89109.

De Renzi, E., Perani, D., Carlesimo, G. A., Silveri, M. C., \& Fazio, F. (1994). Prosopagnosia can be associated with damage confined to the right hemisphere: An MRI and PET study and a review of the literature. Neuropsychologia, 32, 893-902.

Diamond, R., \& Carey, S. (1986). Why faces are and are not special: An effect of expertise. Journal of Experimental Psychology: General, 115, 107-117.

Diana, R. A., Reder, L. M., Arndt, J., \& Park, H. (2006). Models of recognition: A review of arguments in favor of a dual-process account. Psychonomic Bulletin \& Review, 13, 1-21.

Dodson, C. S., \& Schacter, D. L. (2002). When false recognition meets metacognition: The distinctiveness heuristic. Journal of Memory and Language, 46, 782-803.

Eichenbaum, H., Yonelinas, A. P., \& Ranganath, C. (2007). The medial temporal lobe and recognition memory. Annual Review of Neuroscience, $30,123-152$.

Fodor, J. (1983). The modularity of mind: An essay on faculty psychology. Cambridge, MA: MIT Press.

Freire, A., Lee, K., \& Symons, L. A. (2000). The face-inversion effect as a deficit in the encoding of configural information: Direct evidence. Perception, 29, 159-170.

Friedman, N. P., Myake, A., Young, S. E., DeFries, J. C., Corley, R. P., \& Hewitt, J. K. (in press). Individual differences in executive functions are almost entirely genetic in origin. Journal of Experimental Psychology: General.

Hannigan, S. L., \& Reinitz, M. T. (2000). Influences of temporal factors on memory conjunction errors. Applied Cognitive Psychology, 14, 309321.

Hasher, L., \& Zacks, R. T. (1988). Working memory, comprehension and aging: A review and a new view. In G. H. Bower (Ed.), The psychology of learning and motivation: Advances in research and theory (Vol. 22, pp. 193-225). New York: Academic Press.

Jacoby, L. L. (1991). A process dissociation framework: Separating automatic from intentional uses of memory. Journal of Memory and Language, 30, 513-541.

Jacoby, L. L. (1999). Ironic effects of repetition: Measuring age-related differences in memory. Journal of Experimental Psychology: Learning, Memory, and Cognition, 25, 3-22.

Jennings, J. M., \& Jacoby, L. L. (1997). An opposition procedure for detecting age-related deficits in recollection: Telling effects of repetition. Psychology and Aging, 12, 352-361.

Jolliffe, I. T. (2002). Principal component analysis. New York: SpringerVerlag.

Jones, T. C., \& Bartlett, J. C. (2009). When false recognition is out of control: The case of facial conjunctions. Memory \& Cognition, 37, 143-157.

Jones, T. C., Bartlett, J. C., \& Wade, K. A. (2006). Nonverbal conjunction errors in recognition memory: Support for familiarity but not for feature bundling. Journal of Memory and Language, 55, 138-155.

Jones, T. C., \& Jacoby, L. L. (2001). Feature and conjunction errors in recognition memory: Evidence for dual-process theory. Journal of Memory and Language, 45, 82-102.

Kassin, S. M., Tubb, V. A., Hosch, H. M., \& Memon, A. (2001). On the "general acceptance" of eyewitness testimony research: A new survey of experts. American Psychologist, 56, 405-416.

Kelley, C. M., \& Jacoby, L. L. (2000). Recollection and familiarity: Process-dissociation. In E. Tulving \& F. I. M. Craik (Eds.), The Oxford handbook of memory (pp. 215-228). New York: Oxford University Press. 
Kroll, N. E. A., Knight, R. T., Metcalfe, J., Wolf, E. S., \& Tulving, E. (1996). Cohesion failure as a source of memory illusions. Journal of Memory and Language, 35, 176-196.

Lampinen, J. M., Odegard, T. N., \& Neuschatz, J. S. (2004). Robust recollection rejection in the memory conjunction paradigm. Journal of Experimental Psychology: Learning, Memory, and Cognition, 30, 332342

Lindsay, R. C. L., \& Wells, G. L. (1985). Improving eyewitness identifications from lineups: Simultaneous versus sequential lineup presentation. Journal of Applied Psychology, 70, 556-564.

Lustig, C., Hasher, L., \& Zacks, R. T. (2007). Inhibitory deficit theory: Recent developments in a "new view." In D. S. Gorfein \& C. M. MacLeod (Eds.), The place of inhibition in cognition (pp. 145-162). Washington, DC: American Psychological Association.

Macmillan, N. A., \& Creelman, C. D. (2005). Detection theory: A user's guide (2nd ed.). Mahwah, NJ: Erlbaum.

Mandler, G. (1980). Recognizing: The judgment of previous occurrence. Psychological Review, 87, 252-271.

Mayes, A. R., Montaldi, D., \& Migo, E. (2007). Associative memory and the medial temporal lobes. Trends in Cognitive Sciences, 11, 126-135.

McKone, E., Kanwisher, N., \& Duchaine, B. C. (2007). Can generic expertise explain special processing for faces? Trends in Cognitive Sciences, 11, 8-15.

McKone, E., Martini, P., \& Nakayama, K. (2003). Isolating holistic processing in faces (and perhaps other objects). In M. A. Peterson \& G. Rhodes (Eds.), Perception of faces, objects, and scenes: Analytic and holistic processes (pp. 92-119). London: Oxford University Press.

McKone, E., \& Peh, Y. X. (2006). Memory conjunction errors for faces are consistent with configural processing. Psychonomic Bulletin \& Review, $13,106-111$

Melton, A. W. (1967). Individual differences and theoretical process variables: General comments on the conference. In R. N. Gagné (Ed.), Learning and individual differences (pp. 238-252). Columbus, OH: Merrill.

Memon, A., \& Bartlett, J. C. (2002). The effects of verbalisation on face recognition. Applied Cognitive Psychology, 16, 635-650.

Milner, B. (1968). Visual recognition and recall after right temporal-lobe excision in man. Neuropsychologia, 6, 191-209.

Moscovitch, M. (1994). Memory and working with memory: Evaluation of a component process model and comparisons with other models. In D. L. Schacter \& E. Tulving (Eds.), Memory systems 1994 (pp. 269-310). Cambridge, MA: MIT Press.

Moscovitch, M., Winocur, G., \& Behrmann, M. (1997). What is special about face recognition? Nineteen experiments on a person with visual object agnosia and dyslexia but normal face recognition. Journal of Cognitive Neuroscience, 9, 555-604.

Odegard, T. N., \& Lampinen, J. M. (2006). Memory editing: Knowledge, criteria and alignment. Memory, 14, 777-787.

Penrod, S. D., \& Bornstein, B. H. (2007). Generalizing eyewitness reliability research. In R. C. L. Lindsay, D. F. Ross, J. D. Read, \& M. P. Toglia (Eds.), Handbook of eyewitness psychology: Vol. II. Memory for people (pp. 529-556). New York: Psychology Press.

Rakover, S. S. (2002). Featural vs. configurational information in faces: A conceptual and empirical analysis. British Journal of Psychology, 93, $1-30$.

Rapcsak, S. Z. (2003). Face memory and its disorders. Current Neurology and Neuroscience Reports, 3, 394-501.

Rapcsak, S. Z., Nielsen, L., Littrell, L. D., Glisky, E. L., Kaszniak, A. W., \& Laguna, J. F. (2001). Face memory impairments in patients with frontal lobe damage. Neurology, 57, 1168-1175.

Rapcsak, S. Z., Reminger, S. L., Glisky, E. L., Kaszniak, A. W., \& Comer, J. F. (1999). Neuropsychological mechanisms of false facial recognition following frontal lobe damage. Cognitive Neuropsychology, 16, 267292
Reder, L. M., Wible, C., \& Martin, J. (1986). Differential memory changes with age: Exact retrieval versus plausible inference. Journal of Experimental Psychology: Learning, Memory, and Cognition, 12, 72-81.

Reinitz, M. T., Lammers, W. J., \& Cochran, B. P. (1992). Memory conjunction errors: Miscombination of stored stimulus features can produce illusions of memory. Memory \& Cognition, 20, 1-11.

Reinitz, M. T., Morrissey, J., \& Demb, J. B. (1994). Role of attention in face encoding. Journal of Experimental Psychology: Learning, Memory, and Cognition, 20, 161-168.

Roediger, H. L., \& McDermott, K. B. (2000). Distortions of memory. In E. Tulving \& F. I. M. Craik (Eds.), The Oxford handbook of memory (pp. 149-162). New York: Oxford University Press.

Rossion, B. Caldara, R., Seghier, M., Schuller, A.-M., Lazeyras, F., \& Mayer, E. (2003). A network of occipito-temporal face-sensitive areas besides the right middle fusiform gyrus is necessary for normal face processing. Brain, 126, 2381-2395.

Rubin, S. R., Van Petten, C., Glisky, E. L., \& Newberg, W. M. (1999). Memory conjunction errors in younger and older adults: Event-related potential and neuropsychological evidence. Cognitive Neuropsychology, $16,459-488$.

Schacter, D. L. (2001). The seven sins of memory: How the mind forgets and remembers. New York: Houghton Mifflin.

Schacter, D. L., Wagner, A. D., \& Buckner, R. L. (2000). Memory systems of 1999. In E. Tulving \& F. I. M. Craik (Eds.), The Oxford handbook of memory (pp. 627-643). New York: Oxford University Press.

Schiltz, C., \& Rossion, B. (2006). Faces are represented holistically in the human occipito-temporal cortex. NeuroImage, 32, 1385-1394.

Searcy, J. H., \& Bartlett, J. C. (1996). Inversion and processing of component and spatial-relational information in faces. Journal of Experimental Psychology: Human Perception and Performance, 22, 904-915.

Searcy, J. H., Bartlett, J. C., \& Memon, A. (1999). Age differences in accuracy and choosing in eyewitness identification and face recognition. Memory \& Cognition, 27, 538-552.

Sergent, J. (1984). An investigation into component and configural processes underlying face perception. British Journal of Psychology, 75, 221-242.

Sergent, J., \& Signoret, J. L. (1992). Varieties of functional deficits in prosopagnosia. Cerebral Cortex, 2, 375-388.

Squire, L. R., Stark, C. E. L., \& Clark, R. E. (2004). The medial temporal lobe. Annual Review of Neuroscience, 27, 279-306.

Stark, C. E. L., \& Squire, L. R. (2003). Hippocampal damage equally impairs memory for single items and memory for conjunctions. Hippocampus, 13, 281-292.

Steblay, N. (1997). Social influence in eyewitness recall: A meta-analytic review of lineup instruction effects. Law and Human Behavior, 21, 283-298.

Steblay, N., Dysart, J., Fulero, S., \& Lindsay, R. C. L. (2001). Eyewitness accuracy rates in sequential and simultaneous lineup presentations: A meta-analytic comparison. Law and Human Behavior, 25, 459-473.

Tanaka, J. W., \& Farah, M. J. (1993). Parts and wholes in face recognition. Quarterly Journal of Experimental Psychology, 46A, 225-245.

Tanaka, J. W., \& Farah, M. J. (2003). The holistic representation of faces. In M. A. Peterson \& G. Rhodes (Eds.), Perception of faces, objects, and scenes: Analytic and holistic processes (pp. 53-74). New York: Oxford University Press.

Tovée, M. J., \& Cohen-Tovée, E. M. (1993). The neural substrates of face processing models: A review. Cognitive Neuropsychology, 10, 505-528.

Tulving, E. (1985). How many memory systems are there? American Psychologist, 40, 385-398.

Underwood, B. J. (1975). Individual differences as a crucible in theory construction. American Psychologist, 30, 128-134.

Underwood, B. J., Kapelak, S. M., \& Malmi, R. A. (1976). Integration of discrete verbal units in recognition memory. Journal of Experimental Psychology: Human Learning and Memory, 2, 293-300. 
Wherry, R. J. (1984). Contributions to correlational analysis. New York: Academic Press.

Yonelinas, A. P. (1997). Recognition memory ROCs for item and associative information: The contribution of recollection and familiarity. Memory \& Cognition, 25, 747-763.

Yonelinas, A. P. (1999). The contribution of recollection and familiarity to recognition and source-memory judgments: A formal dual-process model and an analysis of receiver operating characteristics. Journal of Experimental Psychology: Learning, Memory, and Cognition, 25, 1415-1434.

Yonelinas, A. P. (2002). The nature of recollection and familiarity: A review of 30 years of research. Journal of Memory and Language, 46, 441-517.

Yonelinas, A. P., \& Parks, C. M. (2006). Receiver operating characteristics (ROCs) in recognition memory: A review. Psychological Bulletin, 133, $800-832$.

Received June 19, 2008

Revision received February 24, 2009

Accepted March 25, 2009 